\title{
A Neural Model of Cerebellar Learning for Arm Movement Control: Cortico-Spino-Cerebellar Dynamics
}

\author{
Jose L. Contreras-Vidal, ${ }^{1}$ Stephen Grossberg, ${ }^{2}$ and Daniel Bullock \\ Department of Cognitive and Neural Systems \\ and Center for Adaptive Systems \\ Boston University \\ Boston, Massachusetts 02215
}

\begin{abstract}
A neural network model of opponent cerebellar learning for arm movement control is proposed. The model illustrates how a central pattern generator in cortex and basal ganglia, a neuromuscular force controller in spinal cord, and an adaptive cerebellum cooperate to reduce motor variability during multijoint arm movements using mono- and bi-articular muscles. Cerebellar learning modifies velocity commands to produce phasic antagonist bursts at interpositus nucleus cells whose feed-forward action overcomes inherent limitations of spinal feedback control of tracking. Excitation of $\alpha$ motoneuron pools, combined with inhibition of their Renshaw cells by the cerebellum, facilitate movement initiation and optimal execution. Transcerebellar pathways are opened by learning through long-term depression (LTD) of parallel fiber-Purkinje cell synapses in response to conjunctive stimulation of parallel fibers and climbing fiber discharges that signal muscle stretch errors. The cerebellar circuitry also learns to control opponent muscles pairs, allowing cocontraction and reciprocal inhibition of muscles. Learning is stable, exhibits load compensation properties, and generalizes better across movement speeds if motoneuron pools obey the size principle. The intermittency of climbing fiber
\end{abstract}

\footnotetext{
${ }^{1}$ Present address: Motor Control Laboratory, Arizona State University, Tempe, Arizona 85287-0404.

${ }^{2}$ Corresponding author.
}

discharges maintains stable learning. Long-term potentiation (LTP) in response to uncorrelated parallel fiber signals enables previously weakened synapses to recover. Loss of climbing fibers, in the presence of LTP, can erode normal opponent signal processing. Simulated lesions of the cerebellar network reproduce symptoms of cerebellar disease, including sluggish movement onsets, poor execution of multijoint plans, and abnormally prolonged endpoint oscillations.

\section{Introduction}

Clinical and experimental data on both oculomotor and skeletomotor systems suggest that an intact cerebellum is necessary for the learning and performance of accurate, rapid movements, especially movements involving multiple degrees of freedom. Systems with multiple, mechanically linked degrees of freedom are difficult to control because the inertial, gravitational, and interaction forces, which must be compensated whenever they do not assist desired motion or stasis, are configuration- and rate-dependent. In very slow movements, there can be sufficient time to use sensory feedback to adjust motor commands before errors caused by uncompensated forces can grow very large. But as movement speeds increase, feedback begins to arrive too late to be useful for on-line control of the current movement. In such cases, accuracy cannot be guaranteed unless motor commands to entire groups of muscles can be timed accurately and scaled prior to the arrival of feedback.

Such considerations, in combination with observations regarding the cerebellum's typical "side-

LEARNING \& MEMORY 3:475-502 @ 1997 by Cold Spring Harbor Laboratory Press ISSN1072-0502/97 \$5.00

$$
\begin{array}{lllllllllllllll} 
& E & A & R & N & I & N & G & \begin{array}{l}
\text { 女 } \\
475
\end{array} & M & E & M & O & R & Y
\end{array}
$$


loop" embedding (Fig. 1, below; Ito 1984) within the sensory-motor system, support a view of the cerebellum as an adaptive controller capable of calibrating parallel feed-forward motor commands that can substitute for slow feedback-based commands and thereby enable high speed and accuracy without iterations (Grossberg and Kuperstein 1986). Relatedly, the cerebellum has been viewed by some as an adaptive calibrator of internal forward models that allow computation of the expected effects of motor commands (Kawato and Gomi 1991; Miall et al. 1996). This application of a cerebellar side-loop would allow substitution of fast, "internal feedback" for slow, sensor-based feedback. These two views are not incompatible (Stein and Glickstein 1992). In both perspectives, the cerebellum learns to substitute a faster, anticipatory process for a slower, reactive one. Also compatible with both approaches is the hypothesis that external feedback, although rendered progressively less important for on-line control as cerebellar learning progresses, retains a critical teaching role; such feedback is ultimately the only reliable source of information regarding the adequacy of the timing and scaling of feed-forward movement commands, or of internal model calibration. At the level of the cerebellar system, these approaches thus postulate that feedback error signals in motor coordinates are converted by the inferior olive into discrete teaching signals, which guide incremental, trial-by-trial adjustments in the cerebellar adaptive weights that control anticipatory actions.

Although supported by a wealth of data, the view of the cerebellum as an adaptive controller capable of using feedback to learn how to substitute a faster for a slower "solution" in repeatable task contexts has not gone unchallenged. Brain stem and spinal cord circuitry apparently provide some basis for task-specific recruitment and control of muscle synergies, and experimental studies of decerebrate/decerebellate animals have produced evidence suggesting that such circuitry has some of the same associative competence widely attributed to the cerebellum (Bloedel and Bracha 1995). Although suggestive of partial redundancy, such studies have not shown that the brain stem/ spinal circuitry affords a large, content-addressable memory for learning and storage of the relatively arbitrary task contexts and action groupings required for a large repertoire of learned skills. Nor has it been shown that such circuitry is capable of learning to generate novel movements whose accurate performance requires that different syner- gists (degrees of freedom) be activated at different times after detection of a context stimulus. In contrast, several features of cerebellar architecture and dynamics qualify it as the best known candidate for such a sensory-motor memory: the cerebellum's side-loop embedding, the large-scale projection of state-specifying afferents to the granule cell layer of the cerebellar cortex, the further fanout from granule cells of the parallel fibers (PFs), and the longterm plasticity of PF-Purkinje synapses-now demonstrated both in vitro and in vivo (e.g., Crepel et al. 1996). Moreover, recent modeling studies indicate how the specialized biochemistry of Purkinje cells, particularly the slowly developing calcium responses of the metabotropic glutamate receptor cascade, can allow the cerebellum to learn timed sequences of motor control signals (Fiala and Bullock 1996; Fiala et al. 1996; Kano 1996).

Analyzing the distinct functions of elements of the cerebellar system requires systematic modeling and simulations. By this we mean simulations in which the model cerebellar system includes all of the major cell types; the model cerebellum's afferent signals are generated by simulated receptors and circuits that are part of the simulation; and its efferent signals affect the simulated motor plant via a simulated spinal circuit. In such a model, all major elements of the cerebellar system can be examined for their contribution, if any, to improved operation of a sensory-motor system whose dynamics are realistic enough to appropriately challenge cerebellar provisions for learning, memory, and performance. This report describes such a model, with a focus on how the cerebellar-olivary sideloop can exert learned control of opponently organized limb spinal circuits and thereby improve the performance of rapid two-joint movements, whose desired form is determined by output from a central pattern generator.

Because the cerebellar model interacts with a two-joint limb moved by sets of opponent muscles, the simulations are pertinent to issues of opponency both within the cerebellar system and in the descending pathways from the cerebellum to the spinal cord. Regarding the latter, there has been great interest in recent years in the role of the red nucleus ( $R N$ ) of the reticular formation and the associated cerebello-rubro-spinal pathway. In many vertebrates, this pathway is a key element in the system for voluntary movement, and its analysis promises to illuminate cerebellar function because the magnocellular division of the $\mathrm{RN}$ is dominated by inputs from the nucleus interpositus (NIP) of

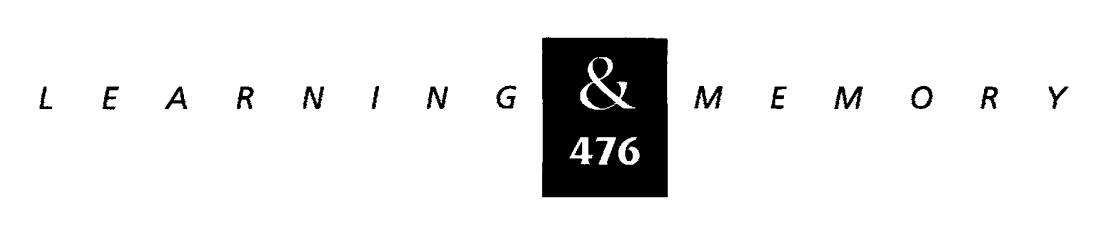


the cerebellum (e.g., Robinson et al. 1987). Much prior modeling work has focused on the role of the NIP/RN in eye blink conditioning (e.g., Bartha et al. 1991; Fiala et al. 1996). The comprehensive skeletomotor simulation strategy followed here refines and elaborates prior treatments of the limbcontrol role of the cerebello-rubro-spinal pathway. Our simulations show a strong advantage, within the opponent muscle system, of the reported dual action- $\alpha$-motoneuron ( $\alpha-\mathrm{MN}$ ) excitation with Renshaw cell inhibition (Henatsch et al. 1986) - of descending signals from the NIP/RN.

Because the cerebellar cortex model includes all major cell types, as well as the interactions implied by their connectivity and sign of action, the results illustrate how interactions in cerebellar cortex may assist rapid switching between the NIP zones that alternately activate opponent muscles during rapid joint rotations. Such rapid switching appears to be necessary for appropriate shaping of the motor command pulses needed to achieve desired acceleration and deceleration of limbs. Taken together with prior results regarding adaptive timing, these results clarify how the cerebellum can contribute to generation of critically timed and shaped, multiphasic burst patterns, which are exhibited by muscles during rapid movement in normal animals but are disordered during cerebellar deactivation (Hore et al. 1991).

The third aspect of our results concerns learning. The model includes a local circuit for signal processing by the olivary nucleus. Its inclusion allowed a demonstration that there is no incompatibility between data indicating that the olive is inherently oscillatory (Llinás 1989), and the hypothesis that the olive's output via climbing fibers constitutes a discrete error feedback-driven teaching signal. The results also show that the climbing fiber (CF) signals produced by the olivary model are suitable for regulating incremental weight adjustments in the cerebellar cortex. In the case studied here, PF-Purkinje cell synapses exhibit both long-term depression (LTD) and long-term potentiation (LTP), depending on whether PF activations do or do not correlate with climbing fiber activations of Purkinje cells (Sakurai 1987). Elsewhere, we have extended the present learning concept to model how the metabotropic glutamate receptor system can enable LTD at the PF-Purkinje cell synapses to adaptively time depression of Purkinje cell firing. This event enables subcortical pathways to read out appropriately timed learned movement gains (Fiala et al. 1996). Because of the opponent form of the model, these learning results also reveal that muscle opponency imposes no constraints on prewiring at the level of the cerebellar cortex other than the constraint that CFs carrying signals of errors from opponent muscle channels should project to distinct parasaggital microzones (Ito 1984). Some aspects of this work have been reported briefly in Bullock et al. (1993a, and unpubl.) and in a dissertation (Contreras-Vidal 1994).

\section{Materials and Methods}

Figure 1 schematizes the components needed to model the cerebellar system as it is embedded within the reaching control system that we studied; but it also exemplifies the typical embedding, in which there is some primary generator of movement, such as a reflex circuit, a locomotor pattern generator, and so on, that sends projections to motoneurons as well as to the cerebellum. The cerebellum in turn sends a projection to the same motoneurons that are addressed by the pattern generator. In addition, there is a feedback signal pathway to one of the olivary nuclei, typically an error feedback signal such as a retinal slip, muscle stretch, unexpected contact, or a signal reporting compensatory action by an error feedback-driven motor command generator. The cerebellum is thus part of a side-loop with respect to the direct projection from primary generator to motoneurons, and the side-loop circuit receives teaching signals generated by residual errors of movement.

The Figure 1 system is shown in greater detail in Figure 2. As the kinematic central pattern generator (CPG), we chose the VITE (Vector Integration To End point) model (Bullock and Grossberg 1988), which embodies key properties of the voluntary, and largely cortical, arm trajectory generation network that outputs to lower brain centers through area 4 (Bullock et al. 1997). The VITE CPG generates desired position and desired velocity commands. As the spinal recipient of commands from both the VITE CPG and the cerebellum, we chose the FLETE model (Bullock and Grossberg 1988, 1991, 1992; Bullock and Contreras-Vidal 1993), an opponent muscle control model of how spinal circuits afford independent voluntary control of joint stiffness and joint position. This model incorporates second-order dynamics, which play a large role in realistic limb movements.

FLETE is an acronym for Factorization of LEngth and TEnsion, which summarizes the most

$$
\text { ………8. }
$$


Figure 1: Cortico-spino-cerebellar model structure: Excitatory pathways from a central pattern generator (VITE) send velocity commands to MNs in the spinal cord as well as mossy fiber collaterals, which may bifurcate and send branches to both the cerebellar cortex and nuclei. The deep cerebellar nuclear cells ultimately project through the RN to MNs and spinal interneurons such as Renshaw cells. Spindle error signals resulting from a comparison of the desired with the actual trajectory project to MNs (not shown) and to the inferior olive, where they act as teaching signals via CFs to the Purkinje cells. These teaching signals regulate LTD at synapses where active PFs contact Purkinje cells. It is hypothesized that for purposes of stability and economy of effort the path for velocity commands through the cerebellum is normally closed by Purkinje inhibition of nuclear cells while the gain of excitatory signals through the MN stage (both from descending commands and from proprioceptive reflexes) is low because of Renshaw inhibition of $\alpha-M N s$. However, LTD opens the gate in the transcerebellar side path and allows transient commands to simultaneously excite MNs and inhibit Renshaw cells. (CPG) Central pattern generator; (NIP) nucleus interpositus cells; (pf) parallel fibers; (cf) climbing fibers; $(\gamma-M N) \gamma$-motoneurons; $(\alpha-M N) \alpha$-motoneurons. Arrow-terminated pathways are excitatory; solid-circle-terminated pathways are inhibitory.

important design principle used to explain why the spinal circuitry evolved to its modern form. For all aspects of motor control, it is critical that the higher nervous system be able to exercise independent control over muscle length and the force or tension developed at that muscle length. The following system of equations describes a subset of the spinal circuitry that can provide this independent control property in a robust way.

The scenario for the simulation studies can be described with reference to Figure 1. Entering on the left is a velocity command (output by the VITE CPG; detail in Fig. 2), which is projected to both cerebellar cortex and to a deep cerebellar nuclear site, here interpreted as a zone within the NIP (Martin and Ghez 1991; Gibson et al. 1985). The NIP projects to and dominates the RN, which in turn excites $\alpha$-MNs and inhibits associated Renshaw cells (Henatsch et al. 1986). However, excitation of motoneurons by this pathway is prevented by Purkinje inhibition of NIP unless PF-signaled contexts from the CPG and spinomuscular system have reliably led to teaching signals from the olive that have induced PF-Purkinje LTD and thereby transiently reduced Purkinje inhibition of the NIP, as has been demonstrated for the analogous case of eye-blink conditioning (for review, see Bullock et al. 1994). The right side of the diagram shows that one proposed teaching signal derives from feedback from muscle spindle organs. Experiments on the stretch reflex (e.g., Matthews 1981) have demonstrated that muscle spindles normally signal the discrepancy (or error) between an intrafusal muscle length setting and the actual extrafusal length. Such discrepancies would also arise naturally during voluntary movement, under conditions of $\alpha-\gamma$ coactivation by a descending desired kinematics signal, if the rate of limb movement/ joint rotation either undershoots or overshoots the desired rate. In summary, to model cerebellar contributions to rapid arm movements, we treat the deep nuclear stage as a normally closed gate which, after learning in particular contexts, will be transiently opened by timed Purkinje cell pausing whenever those contexts recur.

We now present a mathematical specification of the model used to simulate this scenario. The equations used to specify the model mathematically can be divided into three sets: the CPG (VITE) equations, the spinomuscular circuit (two-joint FLETE model) equations, and the cerebellar system equations. Because the first two sets of equations are not a focus of this report, they are introduced with just enough detail to achieve a self-contained exposition. They define the system that is to be assisted by the model cerebellum, which is of pri-

$$
\ldots . . . \cdots
$$




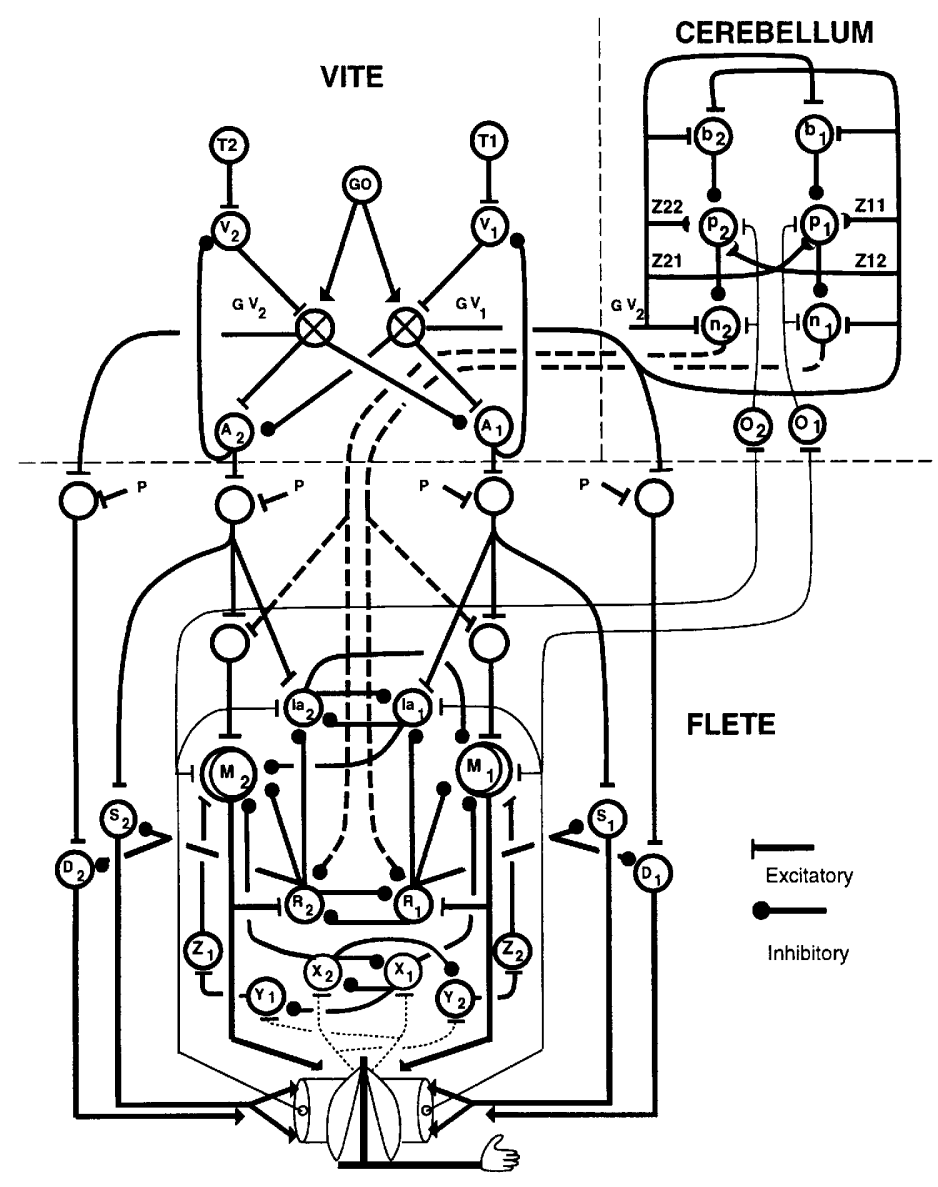

Figure 2: Neural network representation of the neuromuscular control system, including feed-forward cerebellar control. (Top left) The VITE model for variable-speed trajectory generation; (bottom) the FLETE model of the opponently organized spinomuscular system. Dotted lines show feedback pathways from sensors embedded in muscles. The two lateral feedback pathways arise in spindle organs sensitive to muscle stretch and its first derivative. The two medial feedback pathways arise in Golgi tendon organs sensitive to muscle force. Signals $A_{1}$ and $A_{2}$ specify the desired position vector, and the signals $G V_{1}$ and $G V_{2}$ specify the desired velocity vector; signals $T_{1}$ and $T_{2}$ specify the target position vector; signal $P$ scales the level of coactivation, and signal GO scales speed of movement. (Top right) Feedforward cerebellar model computes transient inverse-dynamic signals that excite $M N s$ and modulate the gain in spinal circuits. (b) Basket cell; (P) Purkinje cells; (n) nucleus interpositus cells; $(\mathrm{O})$ inferior olive; (z) long-term memory weights. Paths terminated by solid circles are inhibitory; all others are excitatory. mary concern. On the other hand, Tables 1 and 2 summarize neurobiological evidence for each one of the processing stages of the VITE and FLETE models.

\section{CENTRAL PATTERN GENERATOR: VITE MODEL}

The VITE circuit (Bullock and Grossberg 1988) is a multichannel central pattern generator capable of generating desired arm movement trajectories by smoothly interpolating between initial and final length commands for the synergetic muscles that contribute to a prescribed multijoint movement. The rate of the interpolation, and thus the velocity of movement, is controlled by the product of two signals: a difference vector (DV), which continuously measures the residual vector of muscle length changes needed to reach the final position, and a volitional gating signal, called the GO signal. As shown in the upper part of Figure 2, the DV with components $V_{1}$ and $V_{2}$ is computed in the CPG by subtracting an outflow present position vector (PPV) command with components $A_{1}$ and $A_{2}$ from a target position vector (TPV) command with components $T_{1}$ and $T_{2}$. These relationships lead to the following system.

GO signal dynamics are defined by the sigmoidal functions

$$
G(t)=G_{0} \frac{\left(t-\mathscr{T}_{i}\right)^{2}}{0.5+\left(t-\mathscr{T}_{i}\right)^{2}} u\left[t-\mathscr{T}_{i}\right]
$$

where parameter $G_{0}$ scales the GO signal, $\mathscr{T}_{\mathrm{i}}$ is the onset time of the $i^{\text {th }}$ volitional command, and $u[t]$ is a step function that jumps from 0 to 1 to initiate movement. Difference vector dynamics are defined by

$$
\frac{d}{d t} V_{i}=30\left(-V_{i}+T_{i}-A_{i}\right)
$$

where $T_{\mathrm{i}}$ is the target position command for muscle channel $i, i=1,2$, and $A_{i}$ is the present position command. The difference vector activities

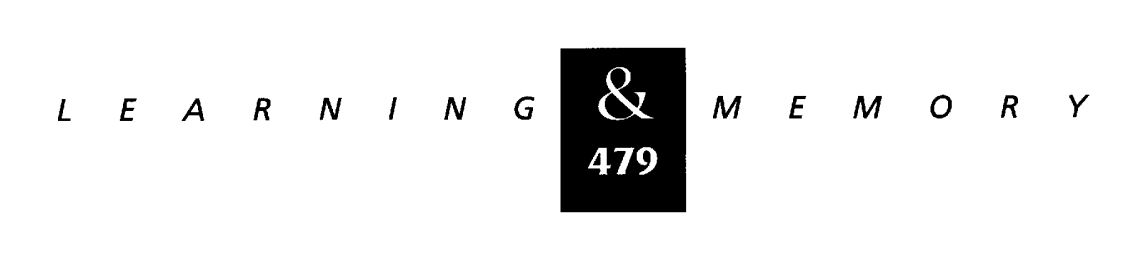


Contreras-Vidal et al.

Table 1: Proposed correspondence between VITE model elements and brain elements

\begin{tabular}{|c|c|c|}
\hline Model element & $\begin{array}{l}\text { Cell type by } \\
\text { physiology }\end{array}$ & References \\
\hline Desired velocity vector (DVV) & $\begin{array}{l}\text { area } 4 \text { phasic movement } \\
\text { time (MT) }\end{array}$ & $\begin{array}{l}\text { Georgopoulos et al. (1982); Fromm et al. } \\
\text { (1984); Kalaska et al. (1989) }\end{array}$ \\
\hline Present position vector (PPV) & area 4 tonic & $\begin{array}{l}\text { Fromm et al. (1984); Kettner et al. } \\
\quad(1988) ; \text { Kalaska et al. (1989) }\end{array}$ \\
\hline Difference vector (DV) & posterior area 5 phasic & $\begin{array}{l}\text { Chapman et al (1984); Crammond and } \\
\text { Kalaska (1989); Kalaska et al. (1990); } \\
\text { Lacquaniti et al. (1995) }\end{array}$ \\
\hline Target position vector (TPV) & area 5 or area $7 b$ & $\begin{array}{l}\text { Robinson and Burton (1980); Anderson } \\
\text { (1987); Dum and Strick (1990); } \\
\text { Lacquaniti et al. (1995) }\end{array}$ \\
\hline GO signal & globus pallidus & $\begin{array}{l}\text { Horak and Anderson (1984a,b); Kato and } \\
\text { Kimura (1992) }\end{array}$ \\
\hline
\end{tabular}

$\left(V_{i}, V_{j}\right)$ are half-wave rectified to generate output signals $\left[V_{i}\right]^{+}$and $\left[V_{j}\right]^{+}$to the next processing stage, where Figure 2 shows that they are multiplied, or gated, by the GO signal to form the vector $\left(G\left[V_{i}\right]^{+}\right.$, $\left.G\left[V_{j}\right]^{+}\right)$. This vector is called the desired velocity vector (DVV) because opponent subtraction of its output signals determine the rate of change of the PPV at the next processing stage:

$$
\frac{d}{d t} A_{i}=G\left[V_{i}\right]^{+}-G\left[V_{j}\right]^{+}
$$

where $\{i, j\}=\{1,2\}$ designate opponent muscle commands. By equation 3 , the PPV integrates the product of opponent GO times DV signals. These equations treat a case where there are are no cerebellar inputs to the CPG, but this need not always be the case.

Prior studies (Bullock and Grossberg 1988, 1991) have demonstrated that the VITE equations can be used to explain a large number of robust kinematic features of voluntary point-to-point movements including smooth, bell-shaped velocity

Table 2: Evidence for connectivity and physiology incorporated in FLETE

\begin{tabular}{|c|c|}
\hline Connection type & References \\
\hline$\alpha-\mathrm{MN}_{i} \stackrel{+}{\rightarrow} R_{i}$ & Renshaw (1941, 1946); Eccles et al. (1954) \\
\hline$R_{i} \rightrightarrows \alpha-M_{i}$ & Renshaw (1941); Eccles et al. (1954) \\
\hline$R_{i} \rightrightarrows l a l N_{i}$ & Hultborn et al. (1971) \\
\hline$R_{i} \rightrightarrows \gamma-\mathrm{MN}_{\mathrm{i}}$ & Ellaway (1968); Ellaway and Murphy (1980) \\
\hline$R_{i} \rightrightarrows R_{j}$ & Ryall (1970); Ryall and Piercey (1971) \\
\hline $\operatorname{laln}_{\mathrm{i}} \rightrightarrows \alpha-\mathrm{MN}_{\mathrm{j}}$ & Eccles and Lundberg (1958); Araki, et al. (1960) \\
\hline $\operatorname{lalN_{i}} \rightrightarrows \operatorname{lalN_{j}}$ & Eccles and Lundberg (1958); Hultborn et al. (1971, 1976); Baldiserra et al. (1987) \\
\hline$l a_{\mathrm{i}}$ fiber $\stackrel{ \pm}{\rightarrow} l a l N_{\mathrm{i}}$ & Hultborn et al. (1971); Baldiserra et al. (1987) \\
\hline$l a_{i}$ fiber $\stackrel{ \pm}{\rightarrow} \alpha-\mathrm{MN}_{\mathrm{i}}$ & Lloyd (1943) \\
\hline$I b I N_{i} \equiv \alpha-M N_{i}$ & Laporte and Lloyd (1952); Eccles et al. (1957); Kirsch and Rymer (1987) \\
\hline$I b I N_{i} \doteq \alpha-M N_{i}$ & Laporte and Lloyd (1952); Eccles et al. (1957) \\
\hline$I b I N_{i} \rightrightarrows I b / N_{j}$ & $\begin{array}{l}\text { Laporte and Lloyd (1952); Eccles et al. (1957); } \\
\text { Brink et al. (1983) }\end{array}$ \\
\hline $\begin{array}{l}\text { Nonspecific } \\
P \stackrel{ \pm}{\rightarrow} \text { spinal } \\
\text { motor pools }\end{array}$ & $\begin{array}{l}\text { Humphrey and Reed (1983); } \\
\text { Deluca (198) }\end{array}$ \\
\hline
\end{tabular}

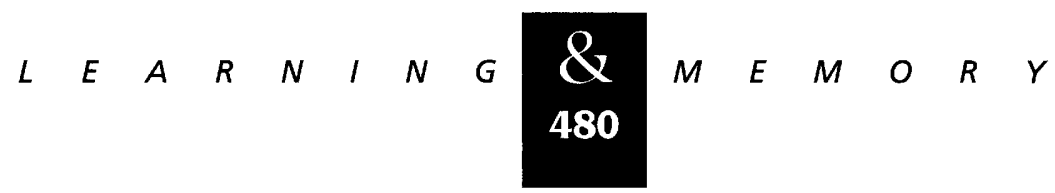


profiles, voluntary speed control that leaves direction and amplitude nearly invariant, peak velocity and acceleration as a function of movement duration, and a Fitts-type speed-accuracy tradeoff. The model's prediction of duration-dependent velocity profiles has been verified experimentally (Nagasaki 1989). Recently, the cell types and connectivity of a self-consistently extended version of the VITE model have been used to simulate neuro-physiological data on six identified cell populations in motor and parietal cortex during a variety of movement tasks (Cisek et al. 1996; Bullock et al. 1997). This model extension uses proprioceptive feedback to adjust perceived position estimates when obstacles are encountered and to compensate for static forces. The processing stages used in the present simulations are shown in Table 1. Also, it has been shown recently how basal ganglia structures can generate a time-varying gating signal that has the properties of the GO signal in the VITE model (Contreras-Vidal and Stelmach 1995).

\section{OPPONENT SPINO-MUSCULAR CIRCUIT: FLETE} MODEL WITH CEREBELLAR INPUTS

This part of the model, shown in the lower part of Figure 2, incorporates an opponent forcegenerating circuit that (1) attempts to implement the CPG movement commands, (2) measures movement errors and returns error signals on a muscle-by-muscle basis when execution is imperfect, and (3) is modulated by descending cerebellar outputs. As shown in Table 2, the FLETE model equations are based on the known neuroanatomy and physiology of the spinomuscular circuits, which include neural, muscular, and sensory parts.

The quadratic force-length relationship of muscle is approximated in the model by

$$
F_{i}=k\left(\left[L_{i}-\Gamma_{i}+C_{i}\right]^{+}\right)^{2}
$$

where indices $i=\{1,2\}$ designate antagonist muscle pairs, $F_{i}$ is muscle force, $L_{i}$ is muscle length, $\Gamma_{i}=$ 20.9 is resting muscle length, and $C_{i}$ is muscle contractile state, thus an activation level, not a length variable. The scaling parameter $k$ was fixed at a value of 1 in the current simulations. The contractile state dynamics are defined by

$$
\frac{d}{d t} C_{i}=\beta_{i}\left[\left(B_{i}-C_{i}\right) M_{i}-C_{i}\right]-\left[F_{i}-\Gamma_{F}\right]^{+}
$$

where the force threshold $\Gamma_{F}=1, M_{i}$ is $\alpha$-MN pool activity in muscle control channel $i, \beta_{i}$ is contractile rate, and $B_{i}$ measures the number of contractile fibers. The origin-to-insertion muscle lengths for opponent monoarticular muscles depend on joint angle $\theta$ as follows:

$$
L_{1}=\sqrt{(\cos \theta)^{2}+(20-\sin \theta)^{2}}
$$

and

$$
L_{2}=\sqrt{(\cos \theta)^{2}+(20+\sin \theta)^{2}}
$$

Equations 6 and 7 indicate that a change of joint angle always implies a length increment in one muscle and a length decrement in its opponent. Limb dynamics for a single joint are specified by

$$
\frac{d^{2}}{d t^{2}} \Theta=\frac{1}{I_{m}}\left(F_{1}-F_{2}+F_{e}-n \frac{d}{d t} \Theta\right)
$$

where $F_{e}$ represents an external force, $F_{i}$ is the force associated with muscle $i, d \Theta / d t$ is angular velocity in radians, $I_{m}$ is the moment of inertia (set to 1 unless specified otherwise), and $n$ is the joint viscosity coefficient (which is used here at a value that allows it to stand in lieu of muscle-related damping; for example, because of the force-velocity characteristic of muscle). The model shows qualitatively similar behavior over a range of viscosities $n$ spanning at least $0.1 \leqslant n \leqslant 0.3$.

Both contraction rate and the number of contractile fibers are known to increase with excitatory input to the $\alpha-\mathrm{MN}$ population, among other factors. This is the size principle of motor unit organization (Hennemann 1957, 1985). Accordingly, contraction rate in equation 5 depends on the level of excitatory input to the $\alpha-\mathrm{MN}$ according to

$$
\beta_{i}=0.05+0.01\left(A_{i}+n_{i}+P+E_{i}\right)
$$

where $A_{i}$ is the descending present position command, $P$ is a coactivation signal (set equal to 0.3 unless specified otherwise), $E_{i}$ is stretch feedback from spindles, and $n_{i}$ is the activity of the NIP/RN zone associated with muscle $i$. Likewise, the number of contractile fibers recruited into force pro- 
duction also depends on the net excitatory drive to the $\alpha-\mathrm{MN}$ :

$$
B_{i}=0.3+3\left(A_{i}+n_{i}+P+E_{i}\right)
$$

Figure 2 shows that axons emerging from motoneuron pools $M_{i}$ send excitatory collaterals to Renshaw cells. The Renshaw population activity is modeled by the membrane equation

$$
\frac{d}{d t} R_{i}=\left(5 B_{i}-R_{i}\right) z_{i} M_{i}-R_{i}\left(0.8+R_{j}+25 n_{i}\right),
$$

where the Renshaw cell recruitment rate $z_{i}$ depended on the level $M_{i}$ of $\alpha-\mathrm{MN}$ activation:

$$
z_{i}=0.05\left(1+M_{i}\right)
$$

The Renshaw population output signal is

$$
R_{i}^{+}=\operatorname{Max}\left[0, R_{i}\right]
$$

which equals $R_{i}$, as $R_{i} \geqslant 0$ by equation 11 . $\operatorname{Max}[0, X]$ means the maximum of 0 or $X$. The $\alpha-\mathrm{MN}$ population activity is also governed by a membrane equation, as are all subsequent model neural populations. Here,

$$
\begin{aligned}
\frac{d}{d t} M_{i}= & \left(\lambda B_{i}-M_{i}\right)\left(A_{i}+n_{i}+P+E_{i}+Z_{j}^{+}\right) \\
& -\left(M_{i}+1.6\right)\left(0.2+R_{i}+X_{i}+I_{j}^{+}\right)
\end{aligned}
$$

where $X_{i}$ is the type $\mathrm{Ib}$ interneuron (IbIN) force feedback (see equation 18) and $Z_{j}^{+}$is a signal dependent on the rate of change of IbIN force feedback in the opponent muscle channel (see equation 20). The $\alpha$-MN population output signal is

$$
M_{i}^{+}=\operatorname{Max}\left[0, M_{i}\right]
$$

Equations 14 and 11 say that descending signals $n_{i}$ from the NIP/RN have an excitatory effect on $\alpha$-MNs and an inhibitory effect on Renshaw cells, as reported by Henatsch et al. (1986). The type $I_{a}$ interneuron (IaIN) population activity is defined by

$$
\frac{d}{d t} I_{i}=\left(10-I_{i}\right)\left(A_{i}+P+E_{i}\right)-\left(I_{i}+1\right)\left(1+R_{i}+I_{j}^{+}\right)
$$

and its output signal is

$$
I_{i}^{+}=\operatorname{Max}\left[0, I_{i}\right]
$$

These equations indicate that model IaINs are reciprocally inhibitory (Hultborn et al. 1971) and are not directly affected by descending signals from the NIP/RN. They are, however, indirectly affected by NIP/RN inhibition of Renshaw cells. The net effect is disinhibition of the IaIN associated with an NIP/RN-activated muscle channel. This effect allows signals from NIP to reinforce reciprocal inhibition.

The IbIN population activity is excited by pathways originating in force-sensitive Golgi tendon organs:

$$
\frac{d}{d t} X_{i}=0.2\left(5-X_{i}\right) F_{i}-X_{i}\left(0.8+0.2 X_{j}\right)
$$

Two other Golgi tendon organ feedback-related activities are a force-derivative related activity:

$$
\frac{d}{d t} Y_{i}=0.2\left(5-Y_{i}\right) F_{i}-Y_{i}\left(1+X_{i}\right)
$$

and an interneuron population activity:

$$
\frac{d}{d t} Z_{i}=0.2\left(5-Z_{i}\right) Y_{i}-Z_{i}
$$

This population's output signal is

$$
Z_{i}^{+}=\operatorname{Max}\left[0, Z_{i}-0.2\right]
$$

Equations $19-21$ and 14 say that the spinal network computes the derivative of force in one channel, delays it, thresholds it, and then uses the resultant signal to excite $\alpha$-MN's in the opponent channel. Such an operation helps brake rapid movements and allows the network to exhibit an inverse myotatic reflex (Bullock et al. 1993b).

The static $\gamma$-MN activity is

$$
\frac{d}{d t} S_{i}=5\left(2-S_{i}\right)\left(A_{i}+P\right)-\left(S_{i}+1.2\right)\left[0.2+0.3 h\left(R_{i}\right)\right]
$$

where $h(w)=\frac{w}{0.3+w}$, and its output signal is

$$
S_{i}^{+}=\operatorname{Max}\left[0, S_{i}\right]
$$

The intrafusal muscle contraction associated with static $\gamma$-MN activation obeys 


$$
\frac{d}{d t} U_{i}=\left(2-U_{i}\right) S_{i}^{+}-U_{i}
$$

The dynamic $\gamma$-MN activity satisfies

$$
\begin{aligned}
\frac{d}{d t} D_{i}= & \left(8-D_{i}\right)\left(100 G\left[V_{i}\right]^{+}+P\right) \\
& -\left(D_{i}+1.2\right)\left[1+100 G\left[V_{j}\right]^{+}+0.5 b\left(R_{i}\right)\right]
\end{aligned}
$$

and its output signal is

$$
D_{i}^{+}=\operatorname{Max}\left[0, D_{i}\right]
$$

The intrafusal muscle contraction associated with dynamic $\gamma$-MN activation is

$$
\frac{d}{d t} N_{i}=0.1\left(2-N_{i}\right) D_{i}^{+}-10 N_{i}
$$

The spindle receptor activation was modeled with

$$
\begin{aligned}
\frac{d}{d t} W_{i}= & \left(2-W_{i}\right)\left(\left[U_{i}+L_{i}-\Gamma_{i}\right]^{+}\right) \\
& +G_{v}\left(\left[N_{i}+\frac{d}{d t} L_{i}\right]^{+}\right)^{0.3}-10 W_{i}
\end{aligned}
$$

where $G_{v}=2$, and the resting length $\Gamma_{i}=20.9$ for these simulations. The stretch feedback signal was a linear function of spindle receptor activation

$$
E_{i}=G_{s} W_{i}
$$

where feedback gain $G_{s}$ was set equal to 1 .

The two-dimensional planar arm simulations used joint receptor feedback from the shoulder joint. For reasons described later, it was assumed that joint receptors activated by shoulder flexion would project only to $\alpha-\mathrm{MN}$ pools that control elbow flexion, and that only those joint receptors activated by shoulder extension would project to $\alpha$-MNs that control elbow extension.

The shape of joint receptors' response functions seems to vary from joint to joint (see Burgess et al. 1982). For example, elbow and wrist joint receptors discharge primarily near the end of the joint range (Millar 1975; Tracey 1979), but those in the hip signal over a large fraction of the working range of this joint (Carli et al. 1979). In addition, some articular receptors do not distinguish be- tween joint flexion or extension, whereas others show responses over roughly half of the joint's range.

Consistent with experimental data (Millar 1975; Tracey 1979) on half-range receptors that fire more strongly as extremes are approached, we modeled the joint receptor's dependence on joint angle in equation 8 with

$$
J_{f}=0.75 \Theta^{1.3} \text { if } \Theta>0 \mathrm{rad}
$$

for shoulder flexion relative to the "zero" angle in the middle of the joint's range and

$$
J_{e}=0.75|\Theta|^{1.3} \text { if } \Theta<0 \mathrm{rad}
$$

for joint receptors activated by shoulder extension relative to the midpoint.

This two-component neural feedback signal specifies joint angle under both static and dynamic (motion) conditions regardless of direction or speed of angular changes. The signal is bounded because of biomechanical limits on the range of joint angle $\theta$. For the two-dimensional simulations, the shoulder joint receptor feedback from the agonist and the antagonist channels was used as additional excitatory inputs to the agonist and antagonist $\alpha$-MN equations for the elbow. Thus, signal $J_{f}$ excited the elbow flexor and signal $J_{e}$ excited the elbow extensor channel. This was accomplished by simply adding these inputs to the excitatory parts of equations otherwise identical to equation 14.

The FLETE model equations have been used in prior reports (Bullock and Contreras-Vidal 1993; Bullock and Grossberg 1989, 1991, 1992) to explain a number of properties of neuromuscular control, including yielding-compensating properties of the size principle of motoneuron recruitment, the ability to voluntarily vary joint stiffness without inadvertently changing posture, and emergence of multiphasic burst patterns in the electromyograms (EMGs) associated with rapid, self-terminated joint rotations.

\section{ADAPTIVE SIDE-LOOP PROCESSING: THE CEREBELLO-OLIVARY MODEL}

The cerebello-olivary model network is schematized in Figure 2 (top right) and in Figure 4, below. Table 3 contains current anatomical and neurophysiological data on the cerebellum that

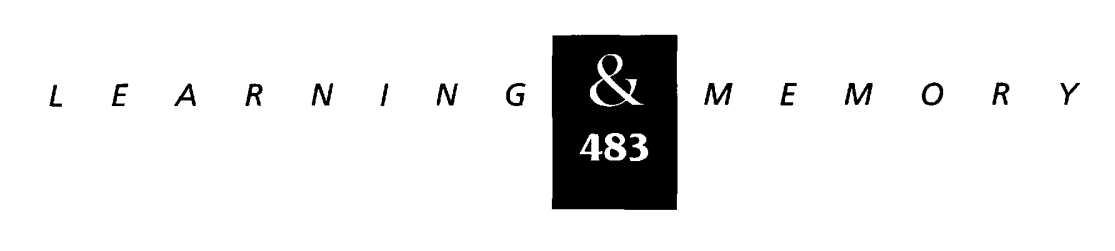


Contreras-Vidal et al.

Table 3: Evidence for connectivity and physiology incorporated in the cerebello-olivary model

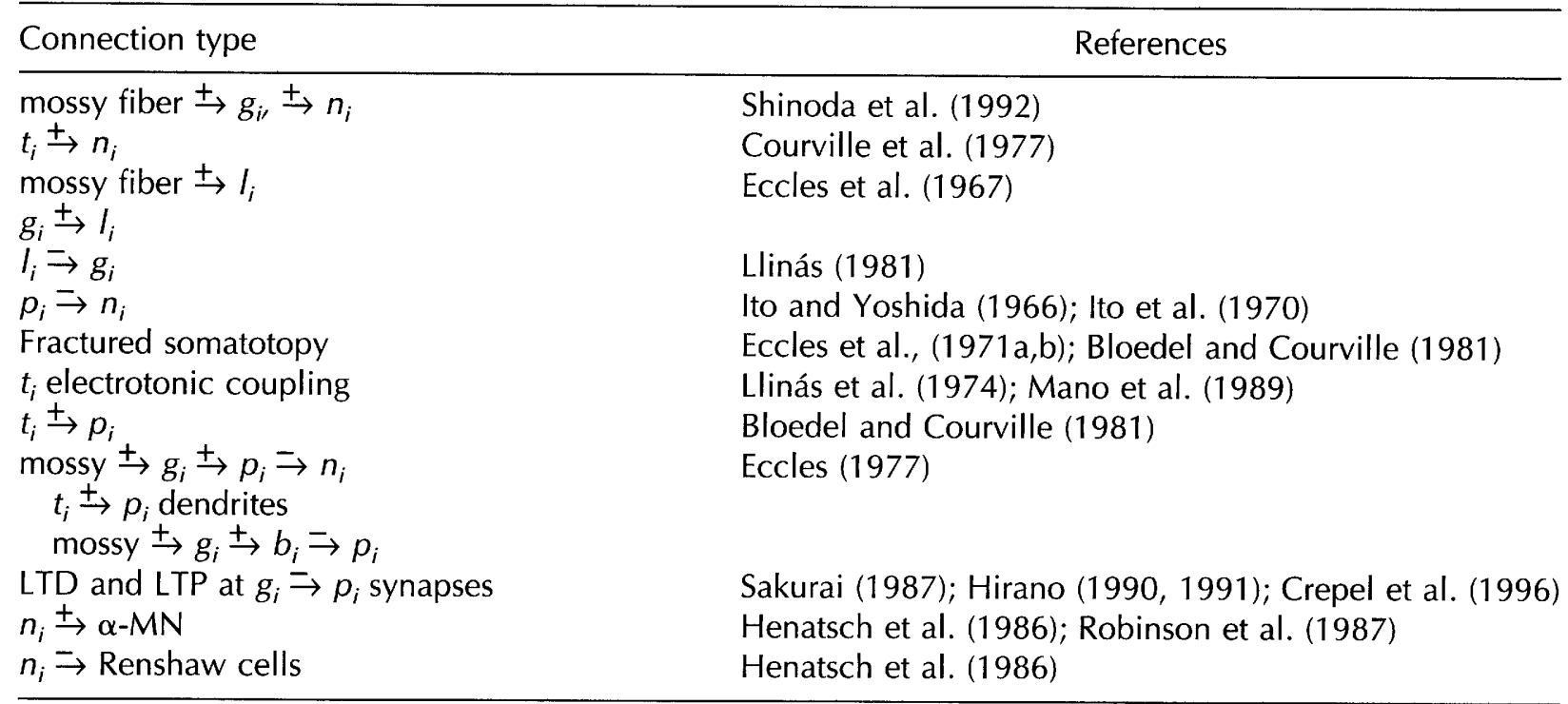

supports the cerebello-olivary network. Purkinje cell activation obeys

$$
\begin{aligned}
\frac{d}{d t} p_{i}= & 2\left[-2 p_{i}+\left(1-p_{i}\right)\right. \\
& \times\left(25 \sum_{k} g_{k} z_{k i}+t_{i}+f\left(p_{i}\right)+0.3\right) \\
& \left.-\left(0.8+p_{i}\right)\left(0.1 p_{j}+b_{i}\right)\right]
\end{aligned}
$$

where $z_{k i}$ is an adaptive weight that multiplies the sampling signal $g_{k}$ carried by the parallel fibers from the $k$ th granule cell to the $i$ th Purkinje cell, $t_{i}$ is a CF teaching signal generated by the $i$ th olivary zone, $f[w]=w^{3} /\left(0.25+w^{3}\right)$ is a self-excitatory sigmoid signal function, and $b_{i}$ is stellate/basket cell inhibitory input. Inhibitory input. Inhibitory stellate/basket-type interneurons were modeled with

$$
\frac{d}{d t} b_{i}=-b_{i}+3\left(2-b_{i}\right)\left(\sum_{k}\left[g_{k}-0.4\right]^{+}\right)
$$

where $g_{k}$ is the activity of the $k$ th granule cell, implies that these cells share parallel fiber receptive fields with the Purkinje cells that they inhibit.

Elements of the NIP/RN stage were modeled with

$$
\begin{aligned}
\frac{d}{d t} n_{i}= & 2\left[\left(-2 n_{i}+\left(1-n_{i}\right)\left(0.2+2500 G\left[V_{i}\right]^{+}\right)\right.\right. \\
& \left.-\left(0.8+n_{i}\right) p_{i}\right] .
\end{aligned}
$$

Thus, the simulated model made no distinction between NIP and RN processing and assumes that each NIP/RN zone receives only one desired velocity signal. An earlier report (Bullock et al. 1994) showed that such specificity could be learned in NIP because climbing fibers (and the teaching signals that they carry) project both to a limited cortical microzone and to the deep nuclear cells that receive inhibition from that region of cerebellar cotrex. Such learning was not modeled in this study, although its outcome was assumed.

Elements of the NIP/RN stage were modeled with

$$
\begin{aligned}
\frac{d}{d t} g_{i}= & 2\left[\left(-2 g_{i}+\left(1-g_{i}\right)\left(0.2+25,000 G\left[V_{i}\right]^{+}\right)\right.\right. \\
& \left.-\left(0.8+g_{i}\right) l_{i}\right]
\end{aligned}
$$

where $G\left[V_{i}\right]^{+}$is a desired velocity input assumed to be projected to the granule by the mossy fiber pathway, and $l_{i}$ is Golgi cell input. This equation indicates that granule cells were excited by desired velocity signals and inhibited by Golgi cells. The Golgi cells were modeled as excited by both desired velocity inputs and granule cell outputs:

$$
\frac{d}{d t} l_{i}=-l_{i}+\left(2-l_{i}\right)\left(25,000 G\left[V_{i}\right]^{+}\left[g_{k}\right]^{+}\right)
$$

This type of granule-Golgi interaction filters the input to produce transient outputs from granule cells with increasing and greater-than-average ex- 
citatory input levels. In these simulations, Golgi modulation served to shorten the duration for which the desired velocity input produced granule output and to differentiate the velocity signal to obtain a phase lead.

The adaptive weights $z_{k i}$ from parallel fibers onto Purkinje cells were adjusted up (LTP) and down (LTD) within a range from 0 to 1 according to the learning law:

$$
100 \frac{d}{d t} z_{k i}=g_{k}\left[30\left(1-z_{k i}\right)-100 t_{i} p_{i} z_{k i}\right]
$$

where $g_{k}$ is the parallel fiber signal from the $k$ th granule cell to the $i$ th Purkinje cell. Term $t_{i}$ is the climbing fiber teaching signal. This equation embodies the hypothesis that LTP occurs whenever PF synapses are active without coincident CF activity, whereas LTD requires that both PF and CF signals be nonzero (Ito 1991; Ito and Karachot 1992; Fiala et al.). In addition, if an antagonist Purkinje cell activity $p_{j}$ inhibits $p_{i}$, as in equation 32 , then learning may not occur in equation 37 even if $t_{i}$ and $\mathrm{g}_{\mathrm{k}}$ are positive. The learning law (equation 37) hereby realizes a type of opponent learning via the voltage-dependent $p_{i}$. If it is empirically the case that the $C F$ burst invariably depolarizes the Purkinje cell, then a learning law without $p_{i}$ would be functionally equivalent.

The CF teaching signal $t_{i}$ was set equal to the output of the inferior olive network. Each musclerelated channel $i$ of this network, indicated by a site $O_{i}$ in Figure 2, was composed of two model neurons: an excitatory projection neuron with activity $t_{i}$ and an inhibitory interneuron with activity $u_{i}$ (Ellias and Grossberg 1975). The olivary projection neuron is inhibited by the interneuron and excited by spindle error feedback signals from the associated muscle. The olivary interneuron is excited by, and slowly tracks the activation level of, the projection neuron. This system generates a phasic excitatory burst whenever the excitatory input from the spindle exhibits a significant increase in amplitude. However, if the input then remains the same, or decreases, the phasic burst will not be repeated, because of the inhibitory feedback to the projection neuron via the interneuron. Thus,

$$
\begin{aligned}
\frac{d}{d t} t_{i}= & -2 t_{i}+\left(1-t_{i}\right)\left(33.3\left[t_{i}-0.4\right]^{+}+E_{i}\right. \\
& -33.3 t_{i}\left[u_{i}-0.4\right]^{+}
\end{aligned}
$$

and

$$
\frac{d}{d t} u_{i}=0.1\left(-u_{i}+t_{i}\right)
$$

where the spindle activation $E_{i}$ is defined by equation 29. By transforming a step increase into a pulse, this circuit improves stability by preventing the path through the olive to the NIP from operating as a continuous negative feedback pathway. It also delimits the time during which the teaching signal acts in cerebellar cortex, which is needed for precise adaptive timing. However, use of recurrent feedback from the slow inhibitory interneuron also makes the olive inherently oscillatory. In the present context, this experimentally observed property (Llinás 1989) is treated as a side effect rather than as a key functional property.

\section{DYNAMICS FORMULATION OF 2 DEGREES OF FREEDOM PLANAR ARM}

Some simulations of the model incorporate equations (Asada and Slotine 1986) for a 2 degree of freedom planar arm (Fig. 3) that were based on parameters of the human arm. Here we replace equation 8 as follows. Assuming moment arms constant and equal to 1 , the net torque produced by the muscles [pectoralis (pec) and posterior deltoid (pd)] acting at the shoulder becomes

$$
\begin{gathered}
F_{p e c}-F_{p d}=\tau_{1}=H_{11} \ddot{\Theta}+H_{12} \ddot{\Phi}-b \dot{\Phi}^{2}- \\
2 b \dot{\Theta} \dot{\Phi}+G_{1}+b_{1} \dot{\Theta}
\end{gathered}
$$

The net torque produced by muscles [biceps (bic) and triceps (tri)] acting at the elbow becomes

$$
\begin{aligned}
F_{b i c}-F_{t r i}= & \tau_{2}=H_{22} \ddot{\Phi}+H_{12} \ddot{\Theta C}+b \dot{\Theta}^{2}+G_{2}+b_{2} \dot{\Phi} \\
H_{11}= & m_{i} l_{c l}^{2}+I_{1}+m_{2}\left[l_{1}^{2}+l_{c 2}^{2}\right. \\
& \left.+2 l_{1} l_{c 2} \cos (\Phi)\right]+I_{2} \\
H_{22}= & m_{2} l_{c 2}^{2}+I_{2} \\
H_{12}= & m_{2} l_{1} l_{c 2} \cos (\Phi)+m_{2} l_{c 2}^{2}+I_{2} \\
b= & m_{2} l_{1} l_{c 2} \sin (\Phi)
\end{aligned}
$$

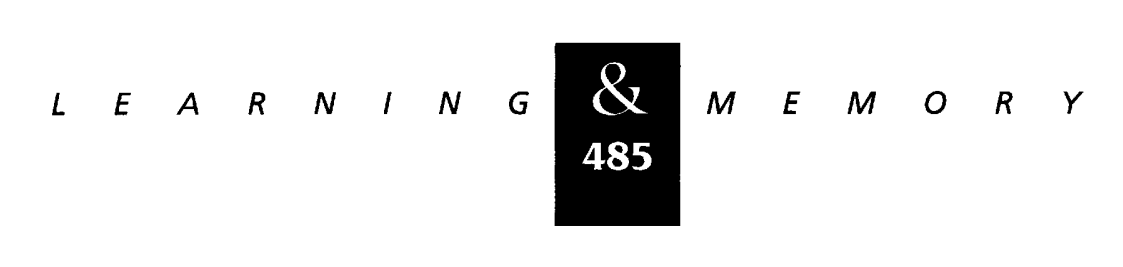


A
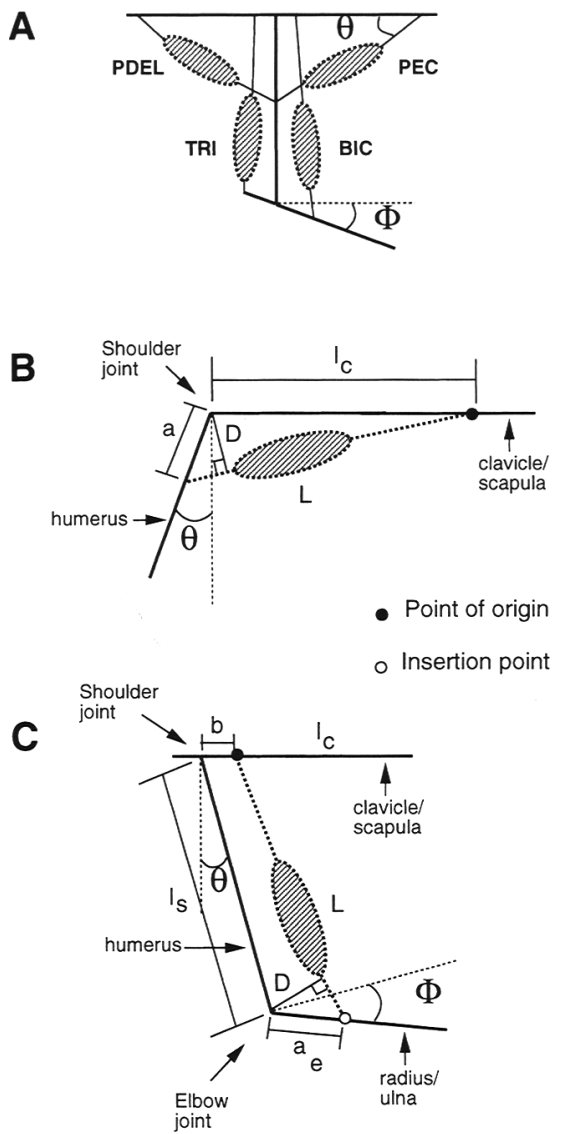

Figure 3: (A) Geometry of origin and insertion points for mono- and biarticular muscles. Monoarticular muscles (PDEL and PEC) cross one joint, biarticular muscles (BIC, TRI) cross two joints. (B) Schematic of PEC muscle geometry (and that of its antagonist PDEL). (C) Schematic of BIC muscle geometry (and that of its antagonistic TRI). ( $\boldsymbol{\theta})$ Shoulder joint angle; $(\Phi)$ elbow joint angle; (a) insertion point for shoulder monoarticular muscle; $\left(\mathrm{a}_{\mathrm{E}}\right)$ insertion point for elbow biarticular 2 muscle; $\left(I_{c}\right)$ distance from clavicle/scapula to shoulder joint; $\left(l_{s}\right)$ length of humerus; (b) muscle origin distance from shoulder joint; (D) normal to the line of pull of muscle.

$$
\begin{aligned}
G_{1}= & m_{1} l_{c 1} g \cos (\Theta)+m_{2} g\left\{l_{c 2} \cos (\Theta+\Phi)\right. \\
& \left.+l_{1} \cos (\Theta)\right\} \\
G_{2}= & m_{2} l_{c 2} m b ; 1 q \cos (\Theta+\Phi)
\end{aligned}
$$

where $\theta$ represents the shoulder joint angle in a planar horizontal arm, $\Phi$ represents the elbow joint angle, $g$ represents the acceleration of gravity along the negative $y$-axis, $b_{1}$ and $b_{2}$ are viscosity coefficients that account for friction at each joint. Though viscosity may be a function of position, velocity, and joint stiffness, in this study it is assumed to be constant. The product of viscosity and angular velocity is important in achieving stability of the limb. We used typical estimates of segment masses $\left(m_{i}\right)$ and segment lengths $\left(l_{i}\right)$ and inertial characteristics from anthropometric data (see Table 4) of Zatsirosky and Seluyanov (1983) and Karst and Hasan (1991).

In our simulations, the shoulder and the elbow are restricted to one rotational degree of freedom (flexion-extension). The muscles whose actions are modeled in these simulations are listed in Table 5 and depicted in Figure 3: (PDEL) posterior deltoid; (PEC) pectoralis major; (BIC) biceps; and (TRI) triceps. To compute muscle lengths, we assumed rotary joints affected by two opponent muscles (i.e., agonist and antagonist), each of which is inserted in the moving segment distal to the axis of rotation. The distance from muscle origin to the axis of rotation was given by $l_{j}, j=c, s$, (see Table 1), and the midpoint of the joint's $180^{\circ}$ excursion was stipulated to be at joint angle $\Theta=0^{\circ}$ and $\Phi=0^{\circ}$ for the shoulder and elbow, respectively. Origin-to-insertion muscle lengths are a function of angle $\Theta$ for the single-joint muscles but are functions of $\Theta$ and $\Phi$ for biarticular muscles. It is assumed that the distance, $l_{c v}, i=1,2$, to the center of mass of each segment link equals half of the length of the line joining the two ends of the link.

The PEC and PDEL muscles are single-joint muscles, and their length at aiven rotation can be computed as follows:

$$
L_{p d}=\sqrt{\left[l_{c}+a \sin ()\right]^{2}+a^{2} \cos ^{2}()}
$$

Table 4: Anthropometric parameter values for the upper limb

\begin{tabular}{ll}
\hline Parameter & \multicolumn{1}{c}{ Value } \\
\hline$m_{1}$ & $1.97 \mathrm{~kg}$ \\
$I_{1}$ & $1.3 \times 10^{-2} \mathrm{~kg} / \mathrm{m}^{2}$ \\
$I_{1}=I_{s}$ & $36.0 \mathrm{~cm}$ \\
$m_{2}$ & $1.64 \mathrm{~kg}$ \\
$I_{2}$ & $2.7 \times 10^{-2} \mathrm{~kg} / \mathrm{m}^{2}$ \\
$I_{2}$ & $47.0 \mathrm{~cm}$ \\
$I_{c 1}=I_{1} / 2$ & $18.0 \mathrm{~cm}$ \\
$I_{c 2}=I_{2} / 2$ & $23.5 \mathrm{~cm}$ \\
$b_{1}, b_{2}$ & $0.3 \mathrm{Nm} . \mathrm{sec} / \mathrm{rad}$ \\
$I_{c}=I_{1} / 2$ & $18.0 \mathrm{~cm}$ \\
$b$ & $1 \mathrm{~cm}$ \\
\hline
\end{tabular}

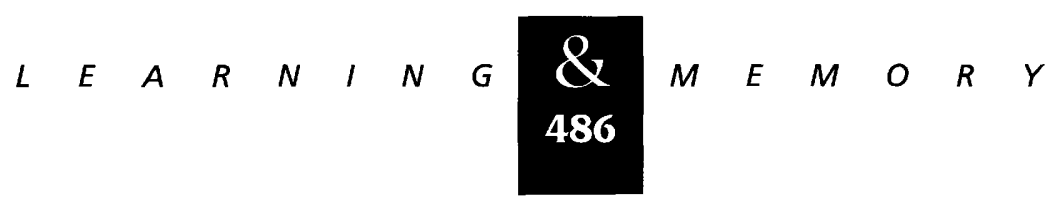


Table 5: Upper arm muscles

\begin{tabular}{llll}
\hline Muscle & \multicolumn{1}{c}{ Origin } & Insertion & Type \\
\hline Biceps-long head (BIC) & glenoid fossa & radius & biarticular \\
Triceps-lateral head (TRI) & fossa of scapula & ulna & biarticular \\
Pectoralis major (PEC) & clavicule & humerus & monoarticular \\
Deltoid-posterior (PDEL) & scapula & humerus & monoarticular \\
\hline
\end{tabular}

where $L_{p d}$ is the length of the PDEL muscle, $l_{1}$ is the distance from the clavicle/spatula to the shoulder joint, $a=10 \mathrm{~cm}$ is the insertion distance from the proximal (shoulder) joint, and $\Theta$ is the angle of rotation centered at $\Theta=0^{\circ}$ (see Fig. 3). For the PEC muscle,

$$
L_{p e c}=\sqrt{\left[l_{c}-a \sin (\Theta)\right]^{2}+a^{2} \cos ^{2}(\Theta)}
$$

Moment arms were assumed to be constant over the whole range of limb movements, and their values were fixed at 1.0. Alexander (1981) has noted that the angle of pennation-namely, the angle at which the muscle fibers pull with maximum force-is approximately a constant between 0.87 and 1.0, which is why the moment arm can be considered constant. In the case of the BIC and TRI, the muscles cross two joints; therefore, the shortening of these muscles is affected by both the elbow joint angle and the shoulder joint angle. We can approximate the length of these muscles as follows (see Fig. 3). In the case of TRI,

$$
L_{t}=\sqrt{\begin{array}{c}
{\left[l_{s}+a_{e} \sin (\Phi)-b \sin (\Theta)\right]^{2}} \\
+\left(a_{e}-b\right)^{2} \cos ^{2}(\Phi)
\end{array}}
$$

where $a_{e}=15 \mathrm{~cm}$ is the muscle insertion distance from the elbow joint, $b=1 \mathrm{~cm}$ is the muscle origin distance from the shoulder joint, $l_{2}$ is the humerus length, $\theta$ is the angle of rotation of the humerus segment with respect to the vertical, and $\Phi$ is the rotation angle of the forearm with respect to the humerus. In the case of the BIC muscle,

$$
l_{b}=\sqrt{\begin{array}{c}
{\left[l_{s}-a_{e} \sin (\Phi)-b \sin (\Theta)\right]^{2}} \\
+\left(a_{e}-b\right)^{2} \cos ^{2}(\Phi)
\end{array}}
$$

Note that the lengths of BIC and TRI cannot be related simply to angle $\Phi$ of the elbow joint. The muscle lengths for biarticulated muscles depend on both the elbow angle and the shoulder angle.

\section{Results}

In this section we present three sets of results. First, we show how adaptive weights evolve in cerebellar cortex when the simulated limb is exercised by a long series of attempted movements. We show that weight evolution converged and was such as to establish a reciprocal pattern of function at this level of the system. Thus, the learning rule is both stable and capable of pruning an initial connectivity to increase efficiency. Second, we show that model NIP/RN activity after learning exhibits phasic bursting, with burst amplitude a function of desired movement rate. This result corresponds to observations of cells in the NIP and the magnocellular portion of the $\mathrm{RN}$, which gives rise to the rubro-spinal projection (e.g., Gibson et al. 1985; Martin and Ghez 1991). We also show that both aspects of the dual projection from RN to the spinal cord are important. In particular, the inhibition of Renshaw cells transiently enhances the gain of the stretch reflex (Hultborn et al. 1979; Henatsch et al. 1986) and prevents premature truncation of $\alpha$-MN bursts needed to launch and brake rapid movements. Third, we show that the model learns to substantially improve the execution of a twojoint movement by compensating for mechanical interactions between the two moving segments. Because this improvement is measured relative to a decerebellate version of the model, which lacked both the model cerebellum and its output to spinal circuits via the rubro-spinal pathway, the improvement is directly attributable to the adaptive cerebello-rubro-spinal system.

\section{LEARNING AND EMERGENCE OF RECIPROCAL PATTERNING IN CEREBELLAR CORTEX}

A major feature of the cerebellar cortex is the asymmetry between the impressive fanout of signals carried by mossy and parallel fibers to widely distributed Purkinje cells and the restricted distri-

$$
\begin{array}{llllllllllllllll} 
& E & A & R & N & I & N & G & \begin{array}{c}
\boldsymbol{Z} \\
\mathbf{4 8 7}
\end{array} & M & E & M & O & R & Y
\end{array}
$$


Contreras-Vidal et al.

bution of signals carried by CFs to bounded sets of Purkinje cells within parasagittal microzones (Ito 1984). This is mimicked in the model by the distribution of desired velocity signals, via the mossygranule-PF pathway, to all Purkinje cells in the model, combined with distribution of each CF to only one Purkinje cell associated with a single NIP/ RN zone and its target muscle; namely, that muscle whose stretch receptors project to the olivary zone giving rise to that $\mathrm{CF}$.

A first question to ask is whether the restricted $\mathrm{CF}$ distribution leads to selective disinhibition of only that NIP/RN site that also receives the desired velocity input and whose projection to spinal cord can help activate the muscle whose contraction is

Figure 4: Opponent cerebellar connectivity before $(A)$ and after $(B)$ learning. All of the synaptic weights between PFs and Purkinje cells were initially set to 1 . During learning, weights in signal pathways $Z_{22}$ and $Z_{11}$ shown in $A$ are diminished through PF to CF LTD, resulting in the connectivity shown in $B$. (C) Model simulations with cerebellar feed-forward learning. (Top) Random gain schedule for the GO signal multiplier during arm movement involving cyclic 25 degree flexion and extension; the GO schedule was randomized over 500 trials (uniform distribution between 1 and 20) to vary the movement speed. (Middle) Learning at PF-Purkinje cell synapses is stable and approaches a steady state after $\sim 400$ movement trials; (bottom) joint position $(\Theta)$ and velocity $(\dot{\Theta})$. traces during the last five movement trials. The joint velocity $\dot{\theta}$ trace shows that the system is able to dynamically track the desired joint velocity $\dot{\Theta}_{d}$. desired. Figure 4 schematizes the network before (Fig. 4A) and after (Fig. 4B) the learning that occurred during a series of single joint flexions and extensions performed by the three component model. Figure $4 \mathrm{C}$ shows that the weight change is initially fast but becomes asymptotic over this series of learning trials. After learning, the PF projection of the velocity signal $G\left[V_{1}\right]^{+}$command in equation 35 to $p_{1}$ in equation 32 has been functionally deleted from the network by LTD but the projection to $p_{2}$ remains. As a result, $G\left[V_{1}\right]^{+}$onset acts at the NIP stage to excite $n_{1}$ and in the cerebellar cortex to inhibit $p_{1}$. The latter effect is achieved because $G\left[V_{1}\right]^{+}$excitation of $p_{2}$ has been removed, whereas the PF projection of $G\left[V_{1}\right]^{+}$con-

A
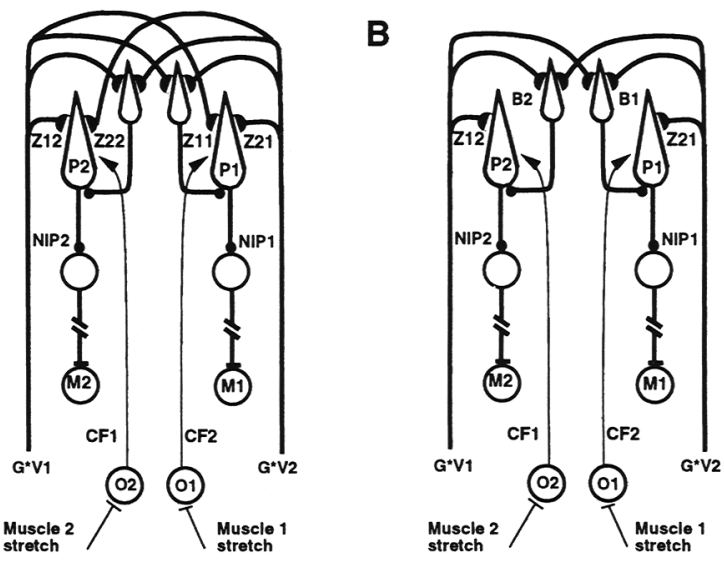

C
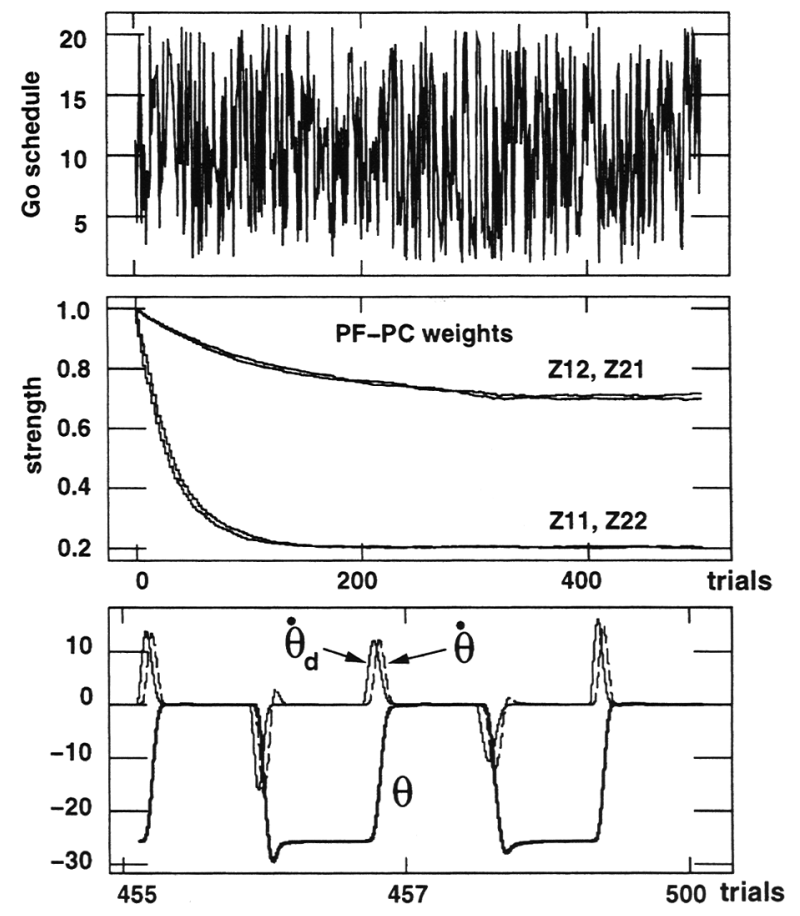
tinues to excite two sources of inhibition to $p_{1}$, namely $b_{1}$ in equation 33 and $p_{2}$ in equation 32 . Such unopposed inhibition of $p_{1}$ leads it to pause, which opens the gate for passage of part of the $G\left[V_{1}\right]^{+}$command through the NIP stage. A recent report on adaptive timing in cerebellum (Fiala et al. 1996) proposed an additional source of Purkinje cell pausing that may complement those simulated here.

BIPHASIC BURST ACTIVITY OF DEEP NUCLEAR CELLS AND EFFECTS OF THE DUAL PROJECTION FROM RN TO SPINAL CIRCUITS

An important benchmark for a cerebellar model is data collected in recent decades on phasic activities of cells in the NIP and in its primary projection target, the magnocellular zone of the RN. In many studies, phasic activity increments in RN have appeared only in cells associated with the agonist muscle (Gibson et al. 1985; Martin and Ghez 1991) and the extended durations of these bursts have led some to interpret the RN signal as a velocity command. In our simulations as well, shown in Figure 5, medium- and low-speed movements produced agonist channel bursts whose duration was similar to that of the entire movement, and therefore might be interpreted as velocity commands (if one were to ignore the actual structure of the model). However, when the speed of desired movement was increased, the agonist
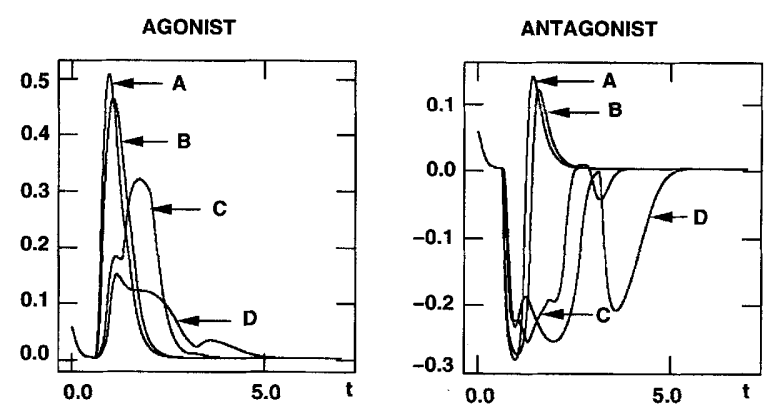

Figure 5: Parametric analysis of the NIP response to movement of different speeds. (Left) Response of the agonist NIP cell; (right) antagonist NIP pool. Condition A corresponds to the faster movement, and condition $D$ corresponds to the slower movement. Two intermediate conditions $(\mathrm{B})$ and $(\mathrm{C})$ are also presented. The input was the desired velocity vector $\left(G\left[V^{+}\right)\right.$signal from the VITE circuit. channel burst at the NIP/RN was shortened significantly, and a following burst emerged in the antagonist channel of the NIP/RN. This bursting pattern was more reminiscent of the multiphasic burst pattern that is observed at the level of the EMG during rapid joint rotations, in which a large braking burst in the antagonist becomes necessary to prevent limb inertia from causing a large overshoot relative to the intended end point of the movement (Lestienne 1979).

The emergence of a braking burst in the model $\mathrm{RN}$ is not surprising, as the role of the cerebellum in the current model is to help generate the torques needed to achieve the desired velocity and position. That is, its role is to compute solutions to inverse dynamics problems. The model thus predicts that if RN activity is examined appropriately during rapid practiced movements, the uniphasic pattern reported in many prior experiments should be replaced with a biphasic pattern distributed across two subpopulations.

The model also clarifies the functional significance of the dual projection from RN to the spinal circuits. In earlier work, it was proposed (Bullock and Grossberg 1989; Akazawa and Kato 1990) that one role of the Renshaw cells is to make the response of the $\alpha-\mathrm{MN}$ pool to excitatory inputs more linear. In the case of an excitatory cocontractive signal sent to both opponent $\alpha-\mathrm{MN}$ pools, such a linearizing effect helps ensure independent control of joint angle and joint stiffness. Such independence is quite important in postural control, when an animal may want to stiffen a joint without changing the difference between the forces acting across the joint.

Movement, however, often requires rapid sequencing through large differences in the forces acting across the joint. Rapid rises of force toward the maximal voluntary force level are impossible unless Renshaw inhibition is curtailed during the rise time. Because one leg of the dual-descending projection from the RN inhibits Renshaw cells, the cerebellum is capable of transiently shifting the $\alpha-\mathrm{MN}$ pools out of the near-linear regime created by Renshaw feedback and into the nonlinear regime made possible by the size principle of $\alpha-\mathrm{MN}$ recruitment. Figure 6 depicts the tracking capabilities of four networks corresponding to the entire circuit of Figure 2 or various subsets thereof. In all cases, the same desired trajectory was generated, for a flexion movement that started from an initially extended joint position. The desired velocity profile appears in each panel as dotted trace c,

$$
\text { , ....... }
$$


Figure 6: VITE-FLETE-CEREBELLUM model simulations for decerebellate network $(A)$, intact system $(B)$, missing inhibitory projections from NIP/RN to Renshaw cells $(C)$, and missing excitatory projections from NIP/RN to $\alpha-M N(D)$. Trace a represents the joint trajectory, $b$ represents the joint velocity, and $c$ represents the desired velocity command.
A DECEREBELLATE

Joint variables

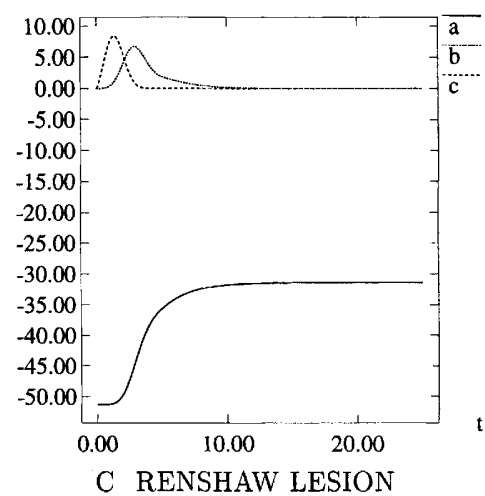

Joint variables

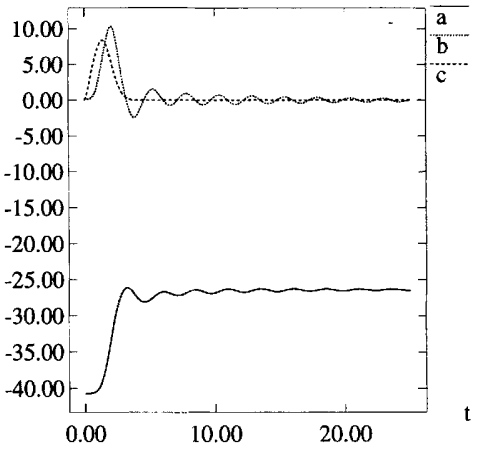

B INTACT

Joint variables

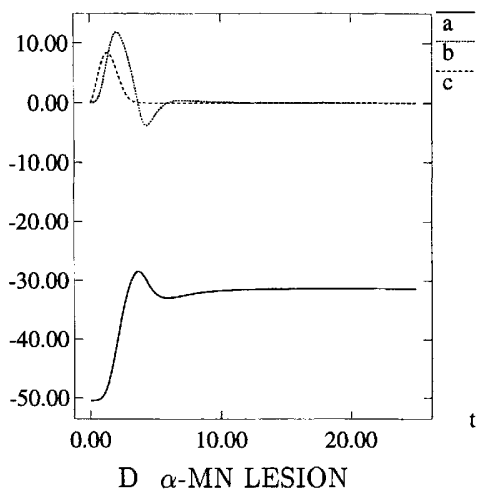

Joint variables

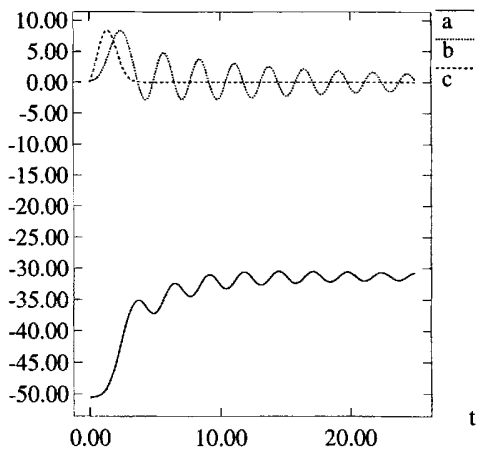

whereas the realized velocity appears as trace a and the realized position as trace $\mathrm{b}$. Figure $6 \mathrm{~A}$ shows the decerebellate case, where the dynamics of the movement are poor compared to the ideal velocity profile. The movement develops slowly and the reaction time is prolonged as seen from the difference between the onsets of desired and actual joint velocities. The joint velocity has a long right tail, and the rise time is slow.

When only the $\mathrm{RN}$ projections to Renshaw cells are eliminated (Fig. 6C), the system shows end-point oscillations that are abnormally prolonged. Cutting the NIP/RN projection to the Renshaw cells removed input related to the phasic and the tonic baseline components of NIP/RN discharge. A side effect is that the equilibrium values of the system are modified, because the Renshaw cells do not receive the inhibitory tonic baseline activity from the NIP/RN cells. When only the RN projections to the $\alpha$-MN pools are eliminated from the system (Fig. 6D), the joint initially undershoots the equilibrium position and oscillates around the end point. This persistent tremor is similar to that seen in cerebellar patients. Without RN inhibition of Renshaw cells, the $\alpha$-MN burst and consequent force development are truncated prior to reaching the levels needed for the desired movement rate.

The effects of specific simulated lesions in the NIP/RN projections to the spinal circuit, corresponding to the simulations shown in Figure 6, C and $\mathrm{D}$, were analyzed in terms of $\alpha$-MN activity and spindle feedback for the network without a NIP/ RN-to-Renshaw projection (Fig. 7A,B) and the network without a NIP/RN-to- $\alpha$-MN pool projection (Fig. 7C,D). Both $\alpha$-MN activity and spindle feedback reflect the oscillatory behavior caused by the cerebellar lesions. Figure 7A shows that the agonist burst is stronger than the antagonist, but there is coactivation of both muscles. A comparison of Figures $7 \mathrm{~B}$ and $8 \mathrm{D}$ indicates that this coactivation results in part from an abnormally large collapse of agonist spindle feedback, which releases the antagonist $\alpha$-MN pool from IaIN-mediated inhibition. The collapse of the agonist spindle feedback is in turn attributable to strong agonist Renshaw inhibition of the agonist $\gamma-\mathrm{MN}$ pool. Thus, the NIP/RN-

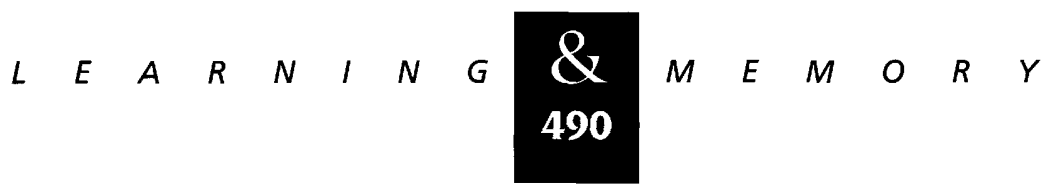


A

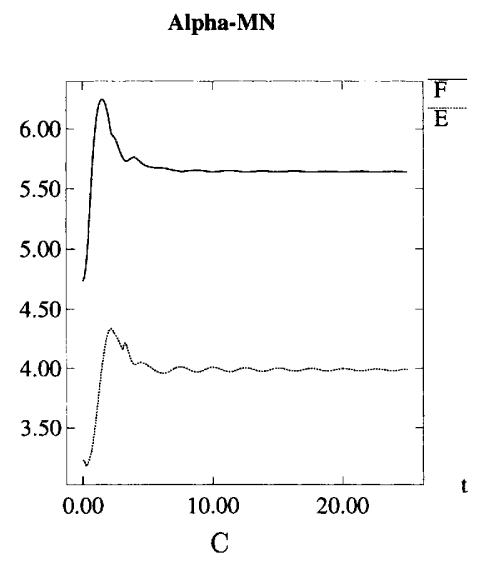

Alpha-MN

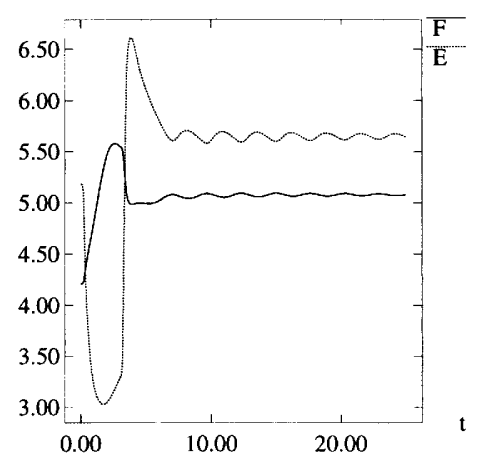

B
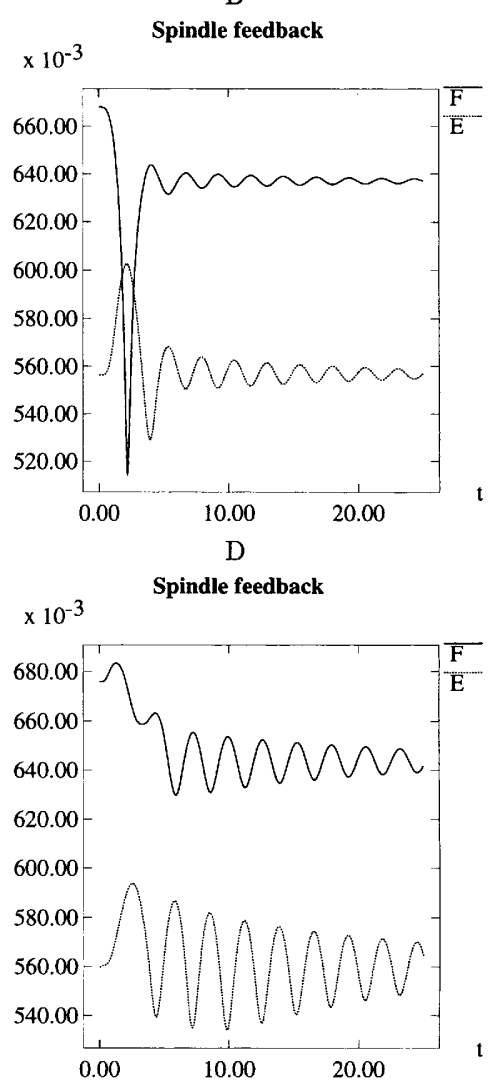

Figure 7: VITE-FLETE-CEREBELLUM model simulations showing $\alpha-M N$ activity and spindle feedback when the projection from NIP/RN to Renshaw cells is eliminated $(A, B)$, and when the NIP/RN-to- $\alpha-M N$ projection is damaged $(C, D)$, in the simulations of Fig. 6. Both simulated lesions produce end-point oscillations, but the latter lesion is more damaging.
to-Renshaw projection normally prevents Renshaw cells from inhibiting $\gamma$-MNs and thereby disrupting the IaIN-mediated pattern of reciprocal activation of agonists and antagonists. Another source of coactivation is the agonist Renshaw inhibition of the antagonist Renshaw pool, which disinhibits the antagonist MN pool.

Figure $7 \mathrm{C}$ shows that the simulated lesion of the NIP/RN-to- $\alpha$-MN projection led to an abnormally small $\alpha$-MN launching burst (cf. Figure $8 \mathrm{C}$ ) and to much less coactivation than in the case of the NIP/RN-to-Renshaw lesion. The delayed antagonist braking burst is nearly normal. This is because the surviving NIP/RN-toRenshaw projection disinhibits the antagonist MN pool at the time when the antagonist spindle feedback signal is growing and the IaIn-mediated inhibition is at a minimum because of collapse of agonist spindle activity. Thus, there is a large transient enhancement of the gain of the antagonist stretch reflex. These effects combine to produce the initial undershoot observed in Figure $6 \mathrm{D}$.

\section{EQUILIBRIA OF THE TWO-DIMENSIONAL PLANAR ARM}

To illustrate the cerebellar opponent learning capabilities using a two-dimensional planar arm with mono- and biarticular muscles, we used two independent single-joint VITE-FLETE systems (Bullock et al. 1993a, and unpubl.). Each FLETE system generates the muscle forces needed to move its corresponding arm segment (e.g., shoulder or elbow joint) by contracting the appropriate muscles according to the planned movement. The biomechanics of the two-dimensional planar arm included the full dynamic equations described earlier (equations 40 and 41), except that the gravitational effects were neglected by assuming a pure horizontal movement; that is, $g=0$ in equations 46 and 47 . Figure 9 depicts the steady-state response of the planar limb controlled by a decerebellate multijoint VITE-FLETE system. Joint angles for the shoulder and elbow as a function of the difference between the descending PPV commands, that is, $A_{1}-A_{2}$ (see Fig. 2 and equations 9 and 10), are shown for each joint.

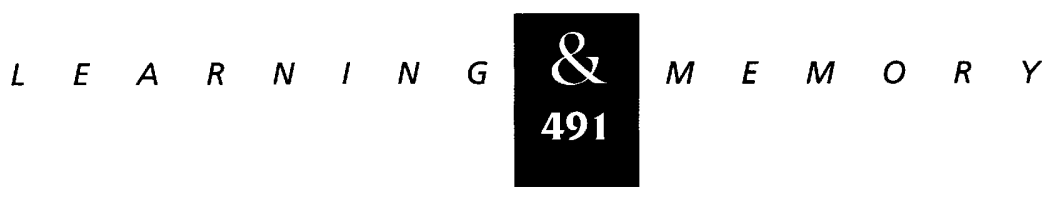


Figure 8: Key variables are depicted from flexor and extensor channels for the intact network simulation of Fig. 6. (A) The net input to the $\alpha-M N$ pools. This input is composed of phasic, burst-like NIP/RN output signals superimposed on ramp-like opponent outputs from the PPV stage of the CPG. The simulated EMG activity in $C$ shows a biphasic patterning of muscle activity (AG followed by an ANT burst), whereas the simulated muscle forces in $B$ represent a triphasic pattern (large $A G$, ANT, small second AG). The reciprocal spindle feedback activities are shown in $D$.
A
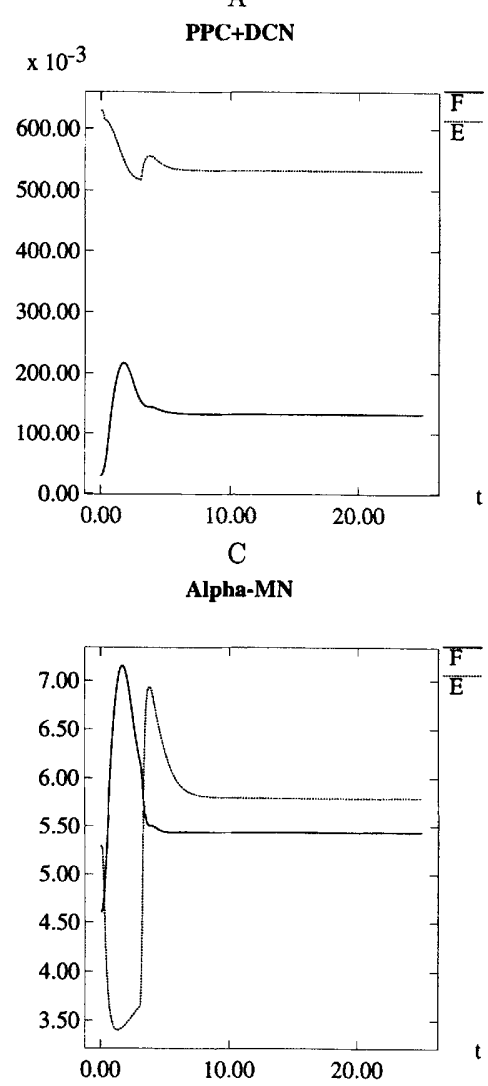

$\mathrm{B}$

Muscle force
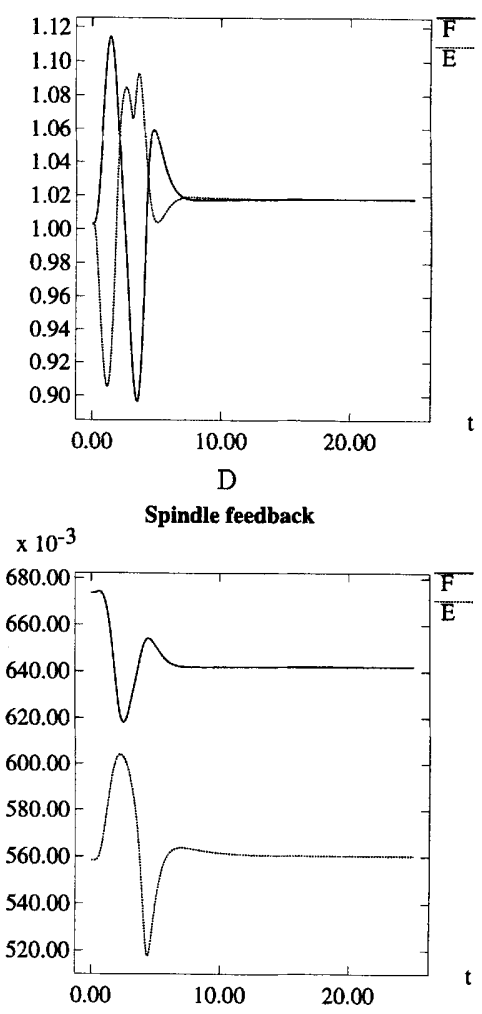

In the absence of external loads, the difference $A_{1}-A_{2}$ would specify a unique equilibrium joint angle in a one-joint FLETE system. Because we have a coupled system here, each set of plots represents a joint angle for a given joint (e.g., shoulder or elbow) versus $A_{1}-A_{2}$, whereas the other joint receives constant input. Positive values of $A_{1}-A_{2}$ mean joint flexion, and negative values mean joint extension. Simulations are shown for a system with and without joint afferent feedback from the shoulder joint to the elbow motoneurons. Figure 9A depicts the steady-state behavior of the shoulder joint angle to the difference $A_{1}-A_{2}$ when the elbow joint received constant input $\left(A_{1}-A_{2}=0.08\right)$. The simulation shows that the shoulder angle is approximately a linear function of the difference $A_{1}-A_{2}$, but the elbow angle rotates (in the extension direction) as the shoulder moves across the work space. The rotation of the elbow joint is increased for higher coactivation values (e.g., the effect is greater for a cocontraction value in equations 9 and 10 of $P=0.4$ than for $P=0.3$ ). This is understandable because the elbow muscles are bi- articular and depend on the shoulder angle. Furthermore, during shoulder flexion, the BIC muscle is unloaded, and an imbalance is produced in favor of the antagonist TRI muscle resulting in the elbow extension. Thus, there was a partial breakdown of the basic FLETE property that the joint angle associated with any choice of $A_{1}-A_{2}$ remains invariant across changes of joint stiffness because of varying the coactivation signal $P$ in equations 9, 10, 14, 16, 22 , and 25 . To compensate, feedback signals denoted by equations 30 and 31 from the shoulder joint receptors act on the elbow motoneuron pools. Joint receptors respond to increased flexion of the shoulder joint by projecting to the flexor $\alpha-\mathrm{MN}$ pool for the elbow, thereby increasing elbow flexion. Because this feedback is position-dependent and monotonic, the outcome is elbow stabilization. This is shown in Figure 9A. In particular, the addition of joint receptor feedback allows the elbow angle to remain constant for a given choice of $A_{1}-A_{2}$ over the full range of shoulder angles. Figure 9B illustrates that the compensation suffices across the work space.

$$
\text { ………………… }
$$


A

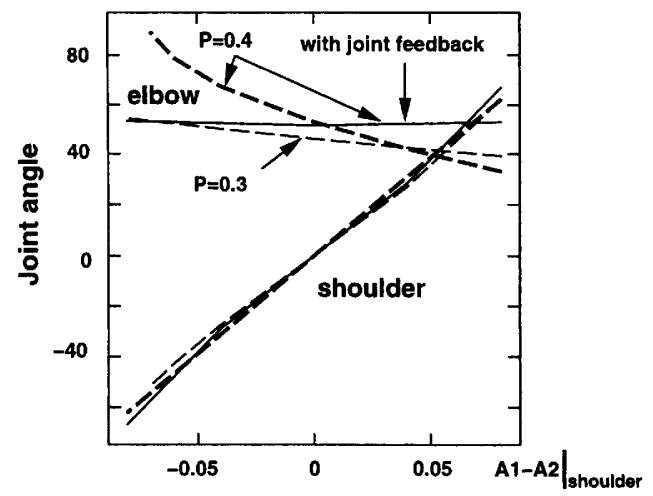

B

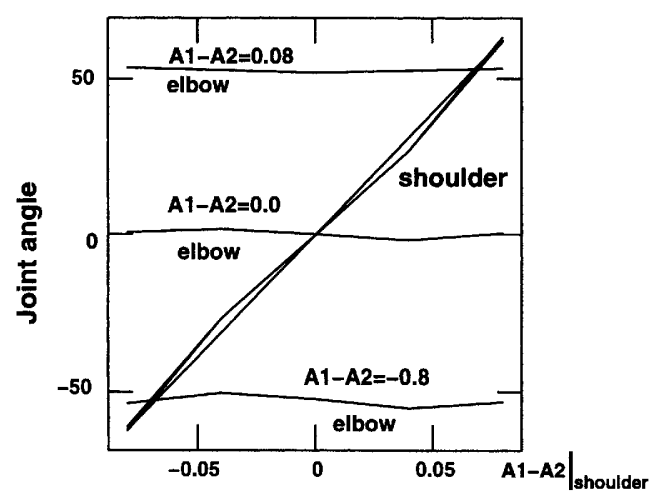

Figure 9: (A) Steady-state response of the planar twojoint limb with and without feedback joint compensation. Without joint feedback, at coactivation settings of either $P=0.4$ or $P=0.3$, elbow angle changes as shoulder angle changes despite constant settings of desired elbow angle commands. Joint receptor feedback from shoulder joint to elbow joint allows the elbow joint angle to remain constant over the full range of shoulder angles if the descending position command for the elbow is held constant. This simulation with hypothesized joint receptor feedback used a coactivation value in equation 9 of $P=0.4$. (B) The linearity is preserved across the work space. In these simulations, the input to the FLETE elbow system was constant during three different input settings corresponding to three regions covering the work space of the planar arm $\left(A_{1}-A_{2}=0.08\right.$, 0 , and -0.08). The input to the FLETE system controlling the shoulder joint was varied from -0.08 to 0.08 .

\section{PERFORMANCE OF CEREBELLATE AND} DECEREBELLATE VERSIONS OF THE MODEL IN THE CONTROL OF A TWO-JOINT ARM MOVEMENT

Cerebellar learning in an opponent motor controller can adaptively discover and form muscle synergies in the case of multijoint arm movements. For a two-joint arm moved by both mono- and biarticular muscles, the feedback learning process can be summarized, as shown in Figure 10. Planar reaching produced by the interplay of two pairs of antagonist muscles admits four cases, as tabulated in Figure10A. Let us assume that the stretch signals generated during two-joint arm movements in the horizontal plane follow a nonoverlapping (alternating, albeit with some degree of coactivation), discrete agonist-antagonist pattern (Fig. 10A). Figure $10 \mathrm{~B}$ shows a representation of the network system before learning, $\mathrm{C}$, during learning, and $\mathrm{D}$, after learning. The rows of panel $\mathrm{B}$ correspond to distinct PFs (denoted by $\mathrm{GrC} 1-\mathrm{GrC} 4$ ), carrying error information for each muscle channel. Each PF projects nonspecifically to all of the Purkinje cells (P1-P4) and cerebellar interneurons (not shown). Initially, the strength of the PF to Purkinje cell connections is set to 1 (Fig. 10B). Activity in PFs and CFs is represented by a box enclosing the activated cells. Each column representing a given Purkinje cell may receive specific activation from its $C F$ (C1-C4). A co-occurrence of PF and CF activation is indicated in Figure $10 \mathrm{C}$ by shading the matrix cell corresponding to the Purkinje axodendritic zone where these two signals could converge. In summary, each Purkinje cell can receive PF inputs from all granule cells and CF inputs only from the unique inferior olive afferent innervating it.

Figure $10 \mathrm{C}$ shows the pattern of activations elicited by conjunctive stimulation of $\mathrm{PF}$ and $\mathrm{CF}$ signals for each case shown in Figure 10A. In case 1, PF signals from $G r C_{1 \mathrm{j}}$ and $G r C_{3 \mathrm{j}}$ arrive at all Purkinje cells as shown by the boxes enclosing them. $\mathrm{CF}$ discharges signaling motor errors in channels $\mathrm{C} 1$ and $\mathrm{C} 3$ also arrive in close temporal and spatial relationship with those PF signals. This would produce LTD of the synapses $G r C_{1 \mathrm{j}}$ to $P 1, G r C_{3 \mathrm{j}}$ to $P 1$, $G r C_{1 \mathrm{j}}$ to $P 3$, and $G r C_{3 \mathrm{j}}$ to $P 3$. After enough trials, encompassing all combinations in Figure 10A, the resulting pattern of connectivity will be like that shown in panel $\mathrm{D}$. In particular, only the weight from the GrC projecting to the opponent Purkinje cell of the same joint will survive LTD. The resulting connectivity matrix shown in Figure 10D can produce the following two outcomes: (1) A single joint stretch (e.g., because of an external load) elicits reciprocal counteraction in the segment of origin as well as stiffening of the adjacent segment; and (2) a two-joint stretch elicits two-joint counteraction.

Using the connectivity matrix in Figure 10D we were able to study the performance of the tripartite model of Figure 2 with and without the cerebellar component during a rapid two-joint movement. Figure 11 shows the state space trajec-

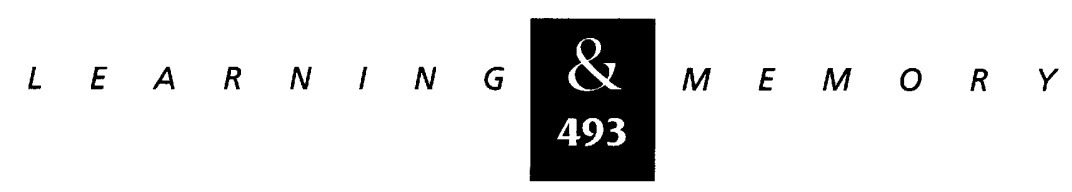


A

\begin{tabular}{|c|c|c|c|c|c|c|}
\hline Case & Shoulder & Elbow & PEC & PDEL & BIC & TRI \\
\hline 1 & Flex & Flex & AG & & $\mathbf{A G}$ & \\
\hline 2 & Ext & Ext & & AG & & $A G$ \\
\hline 3 & Flex & Ext & $\mathbf{A G}$ & & & $\mathbf{A G}$ \\
\hline 4 & Ext & Flex & & AG & AG & \\
\hline
\end{tabular}

B

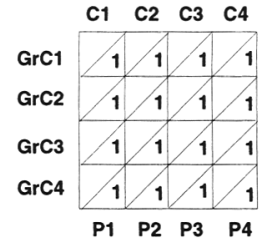

C

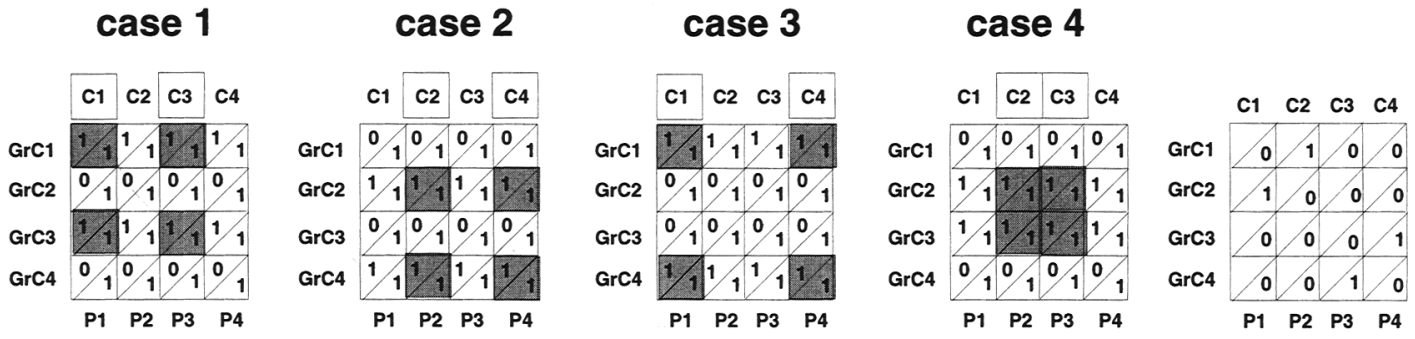

Figure 10: Map representation of the cerebellar connectivity before and after learning. $(A)$ Four possible cases depicting alternating pattern of EMG activation during planar, horizontal, pointing movements. $(B)$ Before learning, all of the synaptic PF $\rightarrow$ Purkinje connections are strong (e.g., set equal to 1). (C) LTD-based cerebellar learning. (D) Synaptic matrix after learning. (C1-C4) Climbing fibers; (P1-P4) Purkinje cells; (GrC1-GrC4) granule cell projections to Purkinje cells (e.g., PFs); (PEC) pectoralis major; (PDEL) posterior deltoid; (BIC) biceps; (TRI) triceps.

tories of elbow and shoulder joints during two movements made with identical CPG settings. One movement was made with the cerebellar component (after learning trials with the two-joint system) and one without. The coordinates for the plotted trajectories are error coordinates: the $x$-axis is joint position error, and the $y$-axis is joint velocity error, with error measured relative to the desired trajectory generated by the CPG. A perfect performance would be represented by a point at the origin, indicating zero velocity and zero position error throughout the trajectory. That neither system approached perfect performance of the desired trajectory indicates that the model is not an optimal controller in its present form. This is not surprising because of the restricted state information available to the cerebellum in these simulations and because of our omission of the spectral timing component of cerebellar learning (Fiala et al. 1996). However, what is more important in the present context is that for each joint the excursion away from the origin was much larger for the decerebellate model than for the model with intact cerebellum. This is a graphic illustration of the error-reductive competence of the simulated cerebellar embedding.

Figure 12 shows the simulated kinematics and EMG activities of the two-joint system with and without cerebellar compensation corresponding to the state space trajectories shown in Figure 11. The decerebellate system was simulated by eliminating the $\mathrm{DV}^{*} \mathrm{G}$ commands to the cerebellar circuitry but maintaining the tonic excitation to both Purkinje and NIP cells, so that the tonic influence of the NIP/RN cells upon spinal cord circuitry was intact, and only the phasic component was disrupted. The movement comprised a $65^{\circ}$ shoulder extension and a $34^{\circ}$ elbow extension. For this simulation, all of the circuitry from Figure 2 was replicated to control two sets of opponent muscles, one monoparticular (P. DEL, PEC), the other biarticular (TRI, BIC). Biomechanical equations were also modified appropriately, as noted above. Heteronymous joint receptor feedback, from the shoulder to elbow motoneurons, was also added to help compensate for static shoulder joint effects at the elbow. The decerebellate simulation shows smaller amplitude muscle activations and more sluggish limb response than in the intact system.

In Figures 6-8, it was shown through simulations of the one-joint case that interpositus lesions degraded the ability of the neuromuscular control system to track the desired velocity of the movement, resulting in increased onset latency, rise time, and settling time. Here, through simulations,

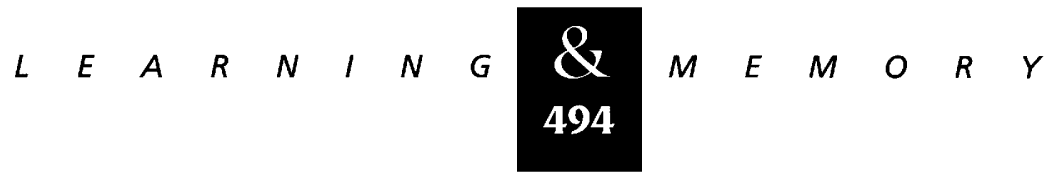


$\mathrm{dE} / \mathrm{dt}$

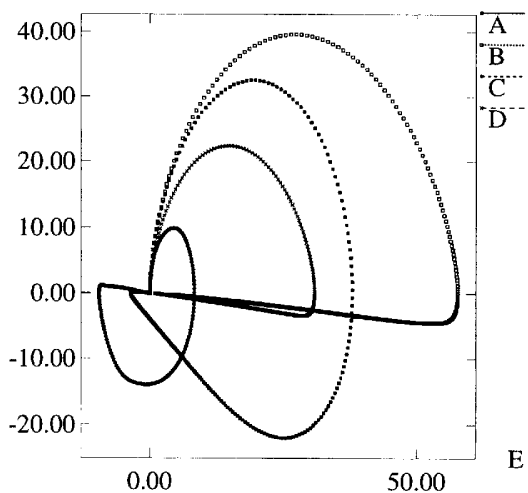

Figure 11: State-space representation of the shoulder and elbow position and velocity tracking for both intact and decerebellate two-joint model simulations. Three variables are shown: The plots show curves of joint position error ( $x$-axis) vs. error derivative ( $y$-axis) for both shoulder and elbow joints over time. $E=\theta-\Theta_{d}$ (shoulder) or $E=\Phi-\Phi_{d}$ (elbow). Plots A (shoulder) and C (elbow) are for the intact system; plots B (shoulder) and D (elbow) correspond to the decerebellate system.

we illustrate the effect of damage to the cerebellar cortex in the two-dimensional system.

Figure 13 shows the effect of a cerebellar lesion on the performance of a multijoint limb flex- ion. This simulation was performed by removing Purkinje cell inhibition from the NIP model cells shown in Figures 1 and 2. The effect of this simulated lesion was to take out the cerebellar processing, except for the direct projections (collaterals) from mossy fibers to NIP cells. This implies that the NIP cells would be disinhibited because of the removal of tonic and phasic Purkinje cell inhibition. In these simulations, it was assumed that the matrix of cerebellar connectivity was already learned (see Fig. 10D); in addition, climbing fibers were not sent to the interpositus neurons. Therefore, these simulations address the role of the mossy fiber projection to the NIP cells alone.

The plots of joint position and velocity of Figure 13B show that the movement evolves slower compared to the intact system simulation shown in panel A. The $\alpha$-MN activities show a higher baseline of activities, which is correlated with the higher level of tonic NIP output (Fig. 14) resulting from removal of Purkinje cell inhibition. Note also that the antagonist motoneuron pool is not inhibited during the early part of the movement and that the antagonist braking bursts are smaller in amplitude. Figure 14 shows that only an agonist burst is generated, albeit of different duration, in the NIP because of the corollary discharge from mossy fi-
DECEREBELLATE
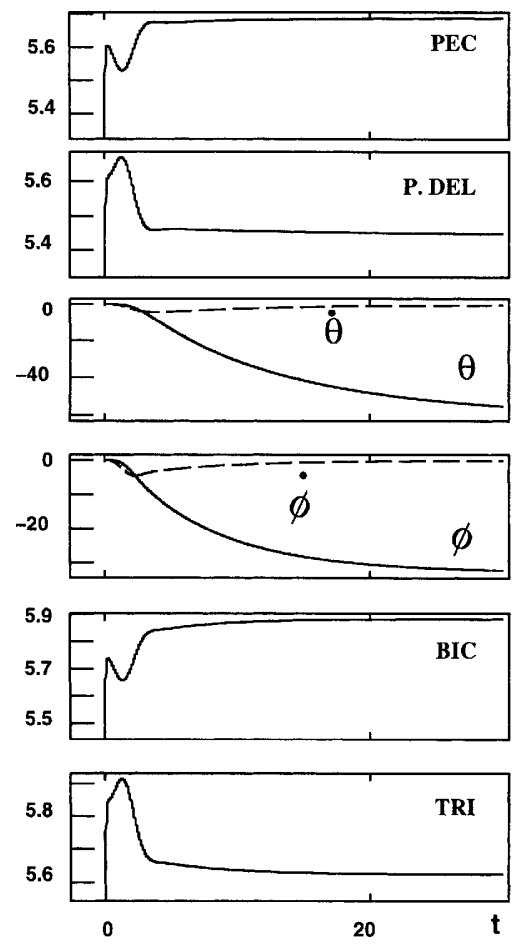

INTACT
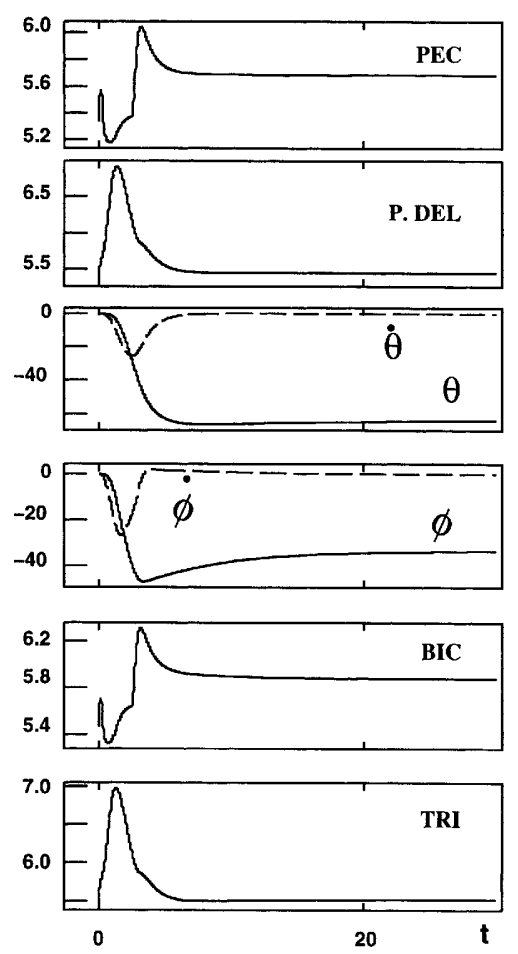

Figure 12: Simulations of the two-dimensional planar arm using two VITEFLETE-CEREBELLUM model systems coupled through joint receptors and the bio-mechanics of the arm. Rows 1, 2, 5, and 6 show dynamics of MN activations associated with four muscles. Rows 3 and 4 show shoulder and elbow kinematics. (PEC) Pectoralis muscle; (P.DEL) posterior deltoid muscle; (BIC) biceps muscle; (TRI) triceps muscle; $\theta$, shoulder joint position; $\dot{\theta}$, shoulder joint velocity; $\Phi$, elbow joint position; $\dot{\Phi}$, elbow joint velocity.

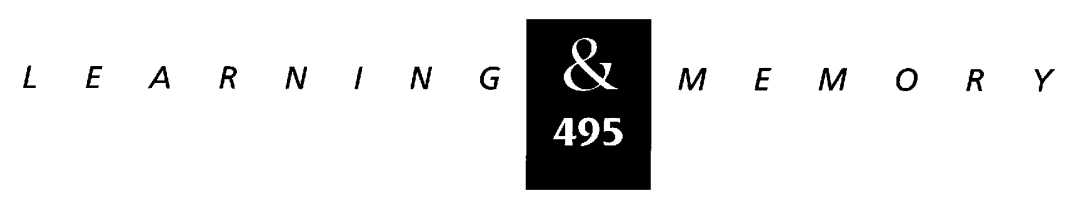


A

Figure 13: Simulation of the dynamics of the two-dimensional planar VITEFLETE-CEREBELLUM system for the intact system $(A)$ and when the cerebellar cortex is damaged $(B)$ (e.g., Purkinje cells no longer inhibit NIP cells). Plots show simulated joint position and velocity for shoulder $(\Theta, \dot{\Theta})$ and elbow $(\Phi, \dot{\Phi})$ joints, as well as simulated mononeuron activities. The Purkinje cell output lesion removes phasic cerebellar disinhibition of NIP cells while also tonically disinhibiting them. This results in higher peak values, reduced ranges, and higher baseline levels of $\alpha-M N$ activity, as well as coactivation of antagonist muscles. The trajectories of the Purkinje-lesioned system show decreased rise times and increased settling times.
INTACT
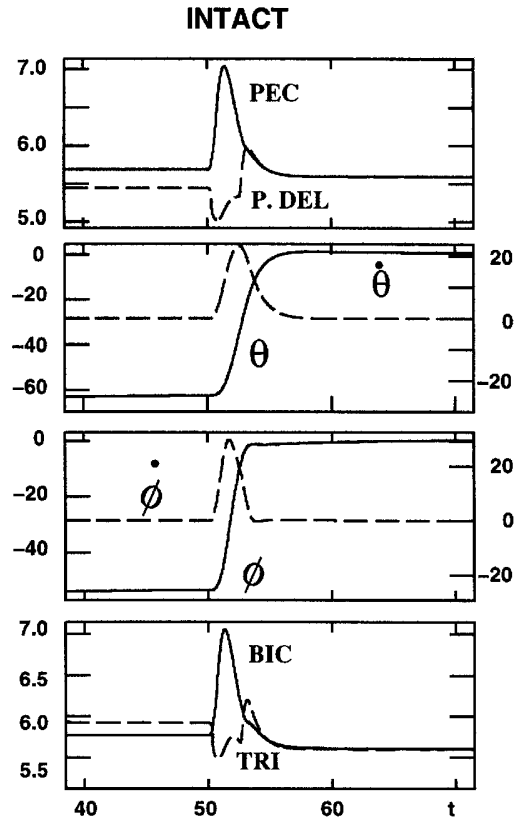

B

PURKINJE CELL LESION
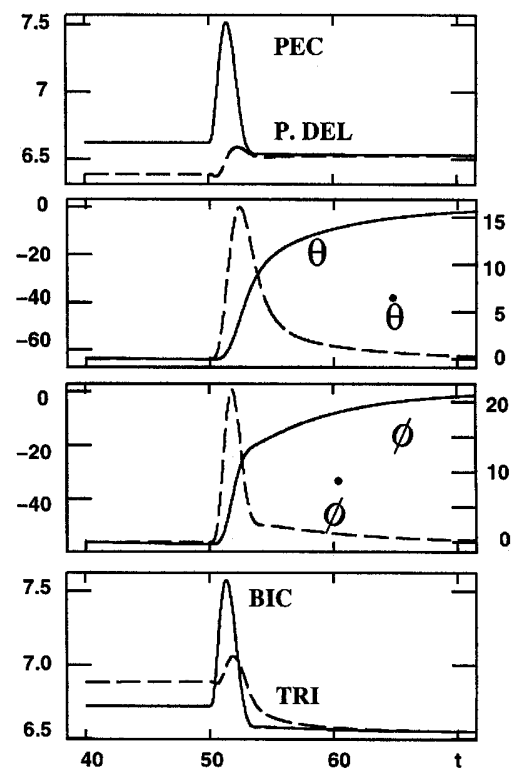

bers from the agonist channel. However, the amplitude of the agonist NIP response is reduced in amplitude after the cerebellar cortex lesion.

These simulations suggest that the cerebellar cortex plays an important role in modulating interpositus nuclear cell discharge, so as to facilitate the initiation and performance of limb movements. In summary, a simulated lesion in the model's cerebellar cortex, without damaging the deep nuclei, shows that although motor performance is degraded, arm movements are still performed, albeit with a slower time course and impaired regularity or smoothness.

\section{Conclusions}

An adaptive, context-dependent, preemptive error reduction mechanism has been proposed to model cerebellar control of descending motor commands and tuning of proprioceptive reflexes during multijoint movements. Specifically, the cerebellar model uses muscle-specific discrete error signals from the inferior olive and nonspecific PF signals from granule cells to learn weight distributions that improve inverse dynamic computations on a trial-by-trial basis.

It is hypothesized that LTD of PF-Purkinje cell synapses, induced by conjunctive stimulation of $\mathrm{PF}$ and CF, and LTP of PF-Purkinje cell synapses, in- duced by repetitive stimulation of PFs alone, can lead to the discovery of opponent muscle synergies by cerebellar circuitry, which enables efficient opponent multijoint muscle control. It is shown that the only requirement for opponent cerebellar control is the specificity of CF projections from the inferior olive to both the cerebellar cortex and the deep nuclear zones that project back through the rubrospinal tract to control the same muscles that

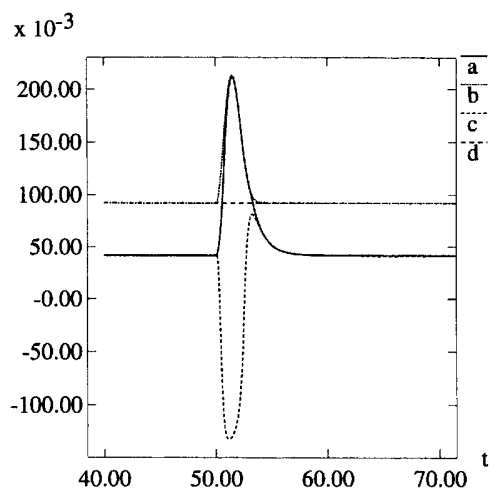

Figure 14: NIP cell discharge for the agonist and antagonist channels before [(a) agonist; (b) antagonist] and after [(c) agonist; (d) antagonist] Purkinje cell lesion. After the Purkinje cell lesion, the baseline of activity of NIP cells increases because of Purkinje cell disinhibition, the duration of the agonist NIP burst is shorter, and no antagonist burst is generated in the other NIP pool.

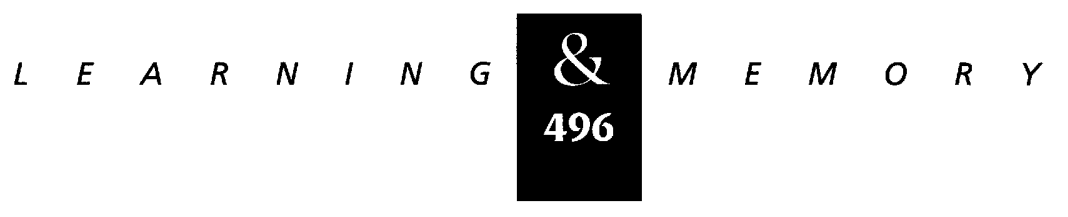


provide afferent inputs to specific inferior olivary zones. Thus, the model incorporates findings indicating that topographical order is maintained through circuits that traverse the olive and associated deep nuclear zones, and shows that this design produces adaptive results when extended to include appropriate somatosensory feedback, in this case, from stretch receptors.

Large-scale simulations of the cerebellar model in interaction with an arm movement cortical central pattern generator and a spinal force controller show that (1) mossy fiber conduction of movement velocity commands from the CPG to both the cerebellar cortex and the associated nucleus NIP/ RN leads to generation of phasic nuclear cell responses whose feed-forward action can greatly improve upon spinal feedback control of tracking, including reduction of oscillations; and (2) phasic NIP/RN excitation of motoneurons, coupled with phasic inhibition of the Renshaw cells associated with those motoneurons, yields better tracking than either operation alone. These results help explain the need for the dual spinal level action of rubral stimulation that was discovered by Henatsch et al. (1986).

OTHER INFLUENCES OF THE CEREBELLAR RUBRAL PATHWAY IN SPINAL CIRCUITRY

Besides reports of direct monosynaptic rubromotoneuronal connections to the forelimb motoneurons of the cat, particularly in the C8-T1 segments (Fujito et al. 1991), and differential effects of stimulation of the cat's RN on lumbar $\alpha$-motoneurons and their Renshaw cells (Henatsch et al. 1986), Hongo et al. (1972b) have shown that stimulation of the RN produces monosynaptic excitatory effects on cells monosynaptically activated, or disynaptically inhibited, from group I muscle afferents and in cells di- or polysynaptically activated from the flexor reflex afferents or exclusively from cutaneous afferents. Hongo et al. (1972a) demonstrated that stimulation of the RN evokes a large dorsal root potential followed by pronounced primary afferent depolarizations in Ib and low threshold cutaneous afferents, and a dual effect on Ia afferent terminals. These monosynaptic effects on Ia afferents or IaINs could contribute to the cerebellar tuning of proprioceptive reflexes and other control loops through changes in the organization, gain, and timing of these reflexes. These effects may be explained using modest ex- tensions to the model proposed in Figure 2, through a more complete analysis of how NIP/RN outputs to spinal cord affect the processing of sensory feedback during arm movement control.

\section{COMPARISON WITH OTHER CEREBELLAR MODELS}

Grossberg and Kuperstein (1986) developed a model of error-based opponent learning of adaptive gain control by the cerebellum. This work built on the cerebellar model of Grossberg (1969), which suggested that both LTD and LTP occurred at the PF-Purkinje cell synapse to learn to control linearly ordered motor components. The early cerebellar model of Marr (1969) assumed that only LTP occurred, whereas that of Albus (1971) invoked LTD at PF synapses with both Purkinje and stellate cells to allow bidirectional weight changes. Smith (1981) proposed how the cerebellar connectivity could allow for agonist-antagonist opponent muscle control. He hypothesized that during cocontraction of antagonist muscles, afferents from each opponent muscle converge on cerebellar inhibitory interneurons to remove the Purkinje cell inhibition of the deep cerebellar nuclei. Furthermore, Smith suggested that by inhibiting groups of deep nuclear cells, Purkinje cells could mediate disfacilitation of motoneuron pools resulting in antagonist muscle relaxation. Recently, Smith (1996) proposed that pairs of Purkinje cells, as in our model and that of Grossberg and Kuperstein (1986), could use reciprocal inhibition or antagonist cocontraction to control agonist-antagonist muscle groups. These models are compatible with the idea that the cerebellum is involved in the learning and coordination of muscle synergies (Thach et al. 1992). Moreover, these models work under the assumption that the command for movement originates outside the cerebellum, that this command provides a reference for computing error feedback signals, and that the cerebellar output projections to the spinal centers (e.g., the rubrospinal tract) form a side path with respect to the descending corticospinal motor pathways (Ito 1984). This is in contrast to models that postulate the cerebellum as an adjustable pattern generator (e.g., Houk et al. 1990, 1993) responsible for primary trajectory generation.

Our opponent cerebellar model for arm movement control extends Smith's (1981, 1996) proposal considerably by demonstrating how an afferent pattern suitable for regulating cocontraction or

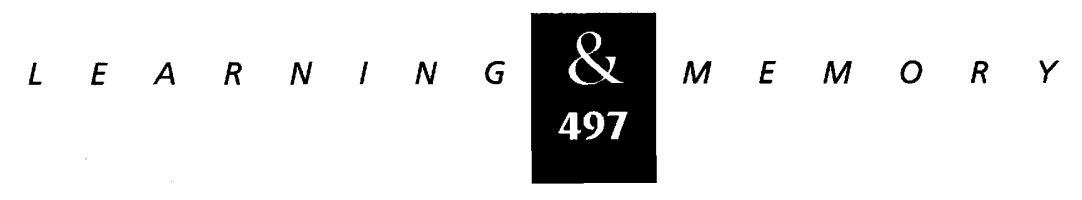


reciprocal inhibition of antagonist muscles can be established by learning because of the spatiotemporal correlation at Purkinje of mossy and CF discharges in the same, but not in opposing or unrelated, channels. This cerebellar learning hypothesis places few demands on a priori wiring for control of opponency in the cerebellum and extends to the control of a large number of muscles acting at several joints. As such, this competence appears to be beyond what can be accomplished by the segmental organizational scheme utilized by spinal stretch reflexes unassisted by learned descending modulation. The proposed cerebellar model allows a generalization of rapid, and in that sense reflex-like, control beyond the one-joint, two-muscle level of organization (Thach et al. 1992; Bullock et al. 1993b).

\section{Acknowledgments}

We wish to thank Diana Meyers for her valuable assistance in the preparation of the manuscript. This work was supported in part by the Monterrey Institute of Technology (Mexico) (J.L.C.) and by the Office of Naval Research (ONR N00014-92-J-1309 and ONR N00014-95-1-0409) (D.B.).

\section{References}

Akazawa, K. and K. Kato. 1990. Neural network model for control of muscle force based on the size principle of motor unit. Proc. IEEE 78: 1531-1535.

Albus, J.S. 1971. A theory of cerebellar function. Math. Biosci. 10: 25-61.

Alexander, R.M. 1981. Mechanics of skeleton and tendons. In Handbook of physiology. Section 1: The nervous system. Vol. II, part 1, Motor control, (ed. F. Plum, V.B. Montcastle, and S.R. Geiger), pp. 17-42. American Physiology Society, Bethesda, MD.

Anderson, R.A. 1987. Inferior parietal lobule function in spatial perception and visuomotor integration. In Handbook of physiology. Section 1: The nervous system, Vol. V, Part 2. Higher functions of the brain (ed. F. Plum, V.B. Mountcastle, and S.R. Geiger), pp. 483-518. American Physiological Society, Bethesda, MD.

Araki, T., J.C. Eccles, and M. Ito. 1960. Correlation of the inhibitory postsynaptic potential of motoneurones with the latency and time course of inhibition of monosynaptic reflexes. J. Physiol. (Lond.) 154: 354-377.

Asada, H. and J.J.E. Slotine. 1986. Robot analysis and control. Wiley-Intersciecne, New York, NY.

Baldissera, F., P. Cavallari, E. Fournier, E. Pierrot-Deseilligny, and M. Shindo. 1987. Evidence for mutual inhibition of opposite la interneurones in the human upper limb. Exp. Brain Res. 66: 106-114.

Bartha, G.T., R.F. Thompson, and M.A. Gluck. 1991. Sensorimotor learning and the cerebellum. In Visual structures and integrated functions (ed. M. Arbib and J. Ewert), pp. 381-396. Springer-Verlag, Berlin, Germany.

Bloedel, J.R. and V. Bracha. 1995. On the cerebellum, cutaneomuscular reflexes, movement and the elusive engrams of memory. Behav. Brain Res. 68: 1-44.

Bloedel, J.R. and J. Courville. 1981. Cerebellar afferent systems. In: Handbook of Physiology. Section 1. The nervous system. Vol. II, part 2, Motor control (ed. F. Plum, V.B. Montcastle, and S.R. Geiger), pp. 735-829. American Physiology Society, Bethesda, MD.

Brink, E., E. Jankowska, D.A. McCrea, and B. Skoog. 1983. Inhibitory interactions between interneurones in reflex pathways from group $l a$ and group $I b$ afferents in the cat. $J$. Physiol. (Lond.) 343: 361-373.

Bullock, D. and J.L. Contreras-Vidal. 1993. How spinal neural networks reduce discrepancies between motor intention and motor realization. In Variability and motor control, (ed. K. Newell and D. Corcos), pp. 183-221. Human Kinetics Press, Champaign, IL.

Bullock, D. and S. Grossberg. 1988. Neural dynamics of planned arm movements: Emergent invariants and speed-accuracy properties during trajectory formation. Psychol. Rev. 95: 49-90.

1989. VITE and FLETE: Neural modules for trajectory formation and tension control. In Volitional action (ed. W. Hershberger), pp. 253-297. North-Holland, Amsterdam, The Netherlands.

1991. Adaptive neural networks for control of movement trajectories invariant under speed and force rescaling. Hum. Movement Sci. 10: 3-53.

1992. Emergence of tri-phasic muscle activation from the nonlinear interactions of central and spinal neural network circuits. Hum. Movement Sci. 11: 157-167.

Bullock, D., J.L. Contreras-Vidal, and S. Grossberg. 1993a. Cerebellar learning in an opponent motor controller for adaptive load compensation synergy formation. In Proceedings of the World Congress on Neural Networks, IV, pp. 481-486. Lawrence Erlbaum, Hillsdale, NJ.

1993b. Equilibria and dynamics of a neural network for opponent muscle control. In Neural networks in robotics (ed. G.A. Bekey and K.Y. Goldberg), pp. 439-457. Kluwer Publishing, Boston, MA.

Bullock, D., J.C. Fiala, and S. Grossberg. 1994. A neural model of timed response learning in the cerebellum. Neural Networks 7: 1101-1114.

Bullock, D., P. Cisek, and S. Grossberg. 1997. Cortical

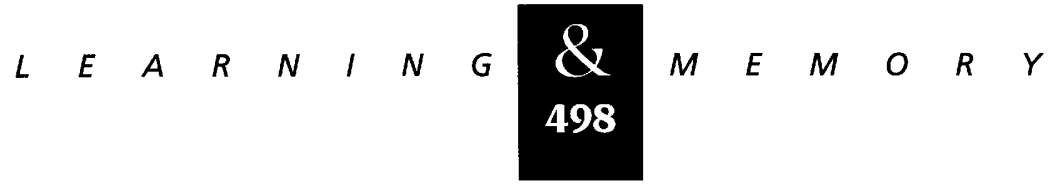


networks for control of voluntary movements under variable force conditions. Technical report CAS/CNS-TR-95-019, Boston University, Boston, MA. Cereb. Cortex (in press).

Burbaud, P., C. Doegle, C. Gross, and B. Bioulac. 1991. A quantitative study of neuronal discharge in areas 5,2 , and 4 of the monkey during fast arm movements. J. Neurophysiol. 66: 429-443.

Burgess, P.R., J.Y. Wei, F.K. Clark, and J. Simon. 1982. Signaling of kinesthestic information by peripheral sensory receptors. Annu. Rev. Neurosci. 5: 171-187.

Carli, G., F. Farabollini, G. Fontani, and M. Meucci. 1979. Slowly adapting receptors in cat hip joint. J. Neurophysiol. 39: 767-778.

Chapman, C.E., G. Spidalieri, and Y. Lamarre. 1984. Discharge properties of area 5 neurones during arm movements triggered by sensory stimuli in the monkey. Brain Res. 309: 63-77.

Cisek, P., S. Grossberg, and D. Bullock. 1996. A cortico-spinal model of reaching and proprioception under multiple task constraints. Technical report CAS/ CNS-TR-96-035. Boston University, Boston, MA.

Collatz, L. 1966. The numerical treatment of differential equations, 3rd ed. Spinger-Verlag, New York, NY.

Contreras-Vidal, J.L. 1994. "Neural networks for motor learning and regulation of posture and movement." Ph.D. dissertation at Boston University. (no. 9334218). University of Michigan, Ann Arbor, MI.

Contreras-Vidal, J.L. and G.E. Stelmach. 1995. A neural model of basal ganglia-thalamocortical relations in normal and parkinsonian movement. Biol. Cybern. 73: 467-476.

Courville, J., J.R. Augustine, and P. Martel. 1977. Projections from the inferior olive to the cerebellar nuclei in the cat demonstrated by retrograde transport of horseradish peroxidase. Brain Res. 130: 405-419.

Crammond, D.J. and J.F. Kalaska. 1989. Neuronal activity in primate parietal cortex area 5 varies with intended movement direction during an instructed-delay period. Exp. Brain Res. 76: 458-462.

Crepel, F., N. Hemart, D. Jaillard, and H. Daniel. 1996. Cellular mechanisms of long-term depression in the cerebellum. Behav. Brain Sci. 19: 347-353.

DeLuca, C.J. 1985. Control properties of motor units. J. Exp. Biol. 115: 125-136.

Dum, R.P. and P.L. Strick. 1990. Premotor areas: Nodal points for parallel efferent systems involved in the central control of movement. In Motor control: Concepts and issues (ed. D.R. Humphrey and D.R. Freund), pp. 383-397. Wiley, London, UK.
Eccles, J. 1977. An instruction-selection theory of learning in the cerebellar cortex. Brain Res. 127: 327-352.

Eccles, R.M. and A. Lundberg. 1958. Integrative pattern of la synaptic actions on motor neurons of hip and knee muscles. J. Physiol. (Lond.) 144: 271-298.

Eccles, J.C., P. Fatt, and K. Koketsu. 1954. Cholinergic and inhibitory synapses in a pathway from motor-axon collaterals to motorneurones. J. Physiol. (Lond.) 126: 524-562.

Eccles, J.C., M. Ito, and J. Szentágothai. 1967. The cerebellum as a neuronal machine. Springer-Verlag, New York, NY.

Eccles, J.C., R.M. Eccles, and A. Lundberg. 1957. The convergence of monosynaptic excitatory afferents on to many different species of alpha motoneurons. J. Physiol. 137: $22-50$.

Eccles, J.C., D.S. Faber, J.T. Murphy, N.H. Sabah, and H. Táboriková. 1971a. Afferent volleys in limb nerves influencing impulse discharges in cerebellar cortex. I. In mossy fibers and granule cells. Exp. Brain Res. 13: 15-35.

Eccles, J.C., D.S. Faber, J.T. Murphy, N.H. Sabah, and H. Táboriková. 1971b. Afferent volleys in limb nerves influencing impulse discharges in cerebellar cortex. II. In Purkinje cells. Exp. Brain Res. 13: 36-53.

Ellaway, P.H. 1968. Antidromic inhibition of fusimotor neurones. J. Physiol. (Lond.) 198: 39P-40P.

Ellaway, P.H. and P.R. Murphy. 1980. A quantitative comparison of recurrent inhibition of $\alpha$ and $\gamma$-motoneurones in the cat. J. Physiol. (Lond.), 315: 43-58.

Ellias, S.A. and S. Grossberg. 1975. Pattern formation, contrast control, and oscillations in the short term memory of shunting on-center off-surround networks. Biol. Cybern. 20: 69-98.

Fiala, J.C. and D. Bullock. 1996. Timing implications of metabotropic mechanisms for cerebellar learning. Behav. Brain Sci. 19: 445-447.

Fiala, J.C., S. Grossberg, and D. Bullock. 1996. Metabotropic glutamate receptor activation in cerebellar Purkinje cells as substrate for adaptive timing of the classically conditioned eye blink response. J. Neurosci. 16: 3760-3774.

Fromm, C., S.P. Wise, and E.V. Evarts. 1984. Sensory response properties of pyramidal tract neurons in the precentral motor cortex and postcentral gyrus of the rhesus monkey. Exp. Brain Res. 54: 177-185.

Fujito, Y., T. Imai, and M. Aoki. 1991. Monosynaptic excitation of motoneurons innervating forelimb muscles following stimulation of the red nucleus in cats. Neurosci. Lett. 127: 137-140.

Georgopoulos, A.P., J.F. Kalaska, R. Caminiti, and J.T. Massey. 1982. On the relations between the direction of

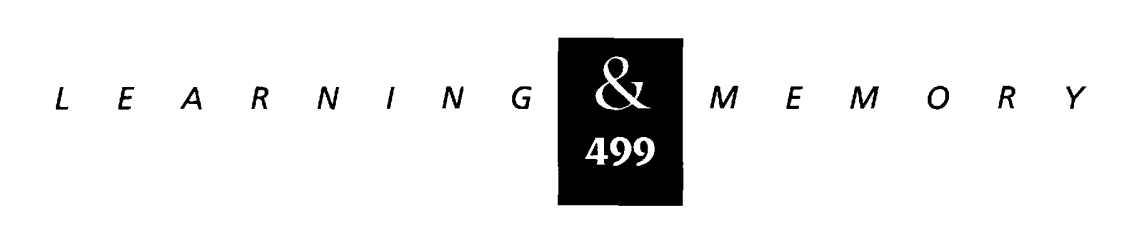




\section{Contreras-Vidal et al.}

two-dimensional arm movements and cell discharge in primate motor cortex. J. Neurosci. 2: 1527-1537.

Gibson, A.R., J.C. Houk, and N.J. Kohlerman. 1985. Relation between red nucleus discharge and movement parameters in trained macaque monkeys. J. Physiol. 358: 551-570.

Grossberg, S. 1969. On learning of spatiotemporal patterns by networks with ordered sensory and motor components. I. Excitatory components of the cerebellum. Stud. Appl. Math. 48: $105-132$.

Grossberg, S. and M. Kuperstein. 1986. Neural dynamics of adaptive sensory-motor control. Pergamon Press, Elmsford, N.Y.

Henatsch, H.D., J. Meyer-Lohmann, U. Windhorst, and J. Schmidt. 1986. Differential effects of stimulation of the cat's red nucleus on lumbar alpha motoneurones and their Renshaw cells. Exp. Brain Res. 62: 161-174.

Henneman, E. 1957. Relation between size of neurons and their susceptibility to discharge. Science 26: 1345-1347.

1985. The size-principle: A deterministic output emerges from a set of probabilistic connections. J. Exp. Biol. 115: 105-112.

Hirano, T. 1990. Depression and potentiation of the synaptic transmission between a granule cell and a Purkinje cell in rat cerebellar culture. Neurosci. Lett. 119: 141-144.

1991. Differential pre- and postsynaptic mechanisms for synaptic potentiation and depression between a granule cell and a Purkinje cell in rat cerebellar culture. Synapse 7: 321-323.

Hongo, T., E. Jankowska, and A. Lundberg. 1972a. The rubrospinal tract III. Effects on primary afferent terminals. Exp. Brain Res. 15: 39-53.

1972b. The rubrospinal tract IV. Effects on interneurons. Exp. Brain Res. 15: 54-78.

Horak, F.B. and M.E. Anderson. 1984a. Influence of globus pallidus on arm movements in monkeys. I. Effects of kainic acid-induced lesions. J. Neurophysiol. 52: 290-304.

1984b. Influence of globus pallidus on arm movements in monkeys. II. Effects of stimulations. J. Neurophysiol. 52: 305-322.

Hore J., B. Wild, and H.C. Diener. 1991. Cerebellar dysmetria at the elbow, wrist and fingers. J. Neurophysiol. 65: 563-571.

Houk, J.C., S.P. Singh, C. Fisher, and A.G. Barto. 1990. An adaptive sensorimotor network inspired by the anatomy and physiology of the cerebellum. In Neural networks for control, Chapter 13 (ed. W.T. Miller, R.S. Sutton, and P.J. Werbos). pp. 301-348. MIT Press, Cambridge, MA.

Houk, J.C., J. Keifer, and A.G. Barto. 1993. Distributed motor commands in the limb premotor network. Trends Neurosci. 16: $27-33$.

Hultborn, H., E. Jankowska, and S. Lindström. 1971. Recurrent inhibition of interneurons monosynaptically activated from group la afferents. J. Physiol. (Lond.), 215: $613-636$.

Hultborn, H., M. Illert, and M. Santini. 1976. Convergence on interneurones mediating the reciprocal la inhibition of motoneurons I. Disynaptic la inhibition of la inhibitory interneurones. Acta Physiol. Scand. 96: 193-201.

Hultborn H., S. Lindström, and H. Wigstrom. 1979. On the function of recurrent inhibition in the spinal cord. Exp. Brain Res. 37: 399-403.

Humphrey, D.R. and D.J. Reed. 1983. Separate cortical systems for control of joint movement and joint stiffness: Reciprocal activation and coactivation of antagonist muscles. In Motor control mechanisms in health and disease (ed. J.E. Desmedt), pp. 347-372. Raven Press, New York, NY.

Ito, M. 1984. The cerebellum and neural control. Raven Press, New York, NY.

_- 1991. The cellular basis of cerebellar plasticity. Curr. Opin. Neurobiol. 1: 616-620.

Ito, M. and L. Karachot. 1992. Protein kinases and phosphatase inhibitors mediating long-term desensitization of glutamate receptors in cerebellar Purkinje cells. Neurosci. Res. 14: 27-38.

Ito, M. and M. Yoshida. 1966. The origin of cerebellar-induced inhibition of Deiters' neurons. I. Monosynaptic initiation of the inhibitory postsynaptic potentials. Exp. Brain Res. 2: 330-349.

Ito, M., M. Yoshida, K. Obata, N. Kawai, and M. Udo. 1970. Inhibitory control of intracerebellar nuclei by the Purkinje cell axons. Exp. Brain Res. 10: 64-80.

Kalaska, J.F., D.A.D. Cohen, M.L. Hyde, and M.J. Prud'homme. 1989. A comparison of movement direction-related versus load direction-related activity in primate motor cortex, using a two-dimensional reaching task. J. Neurosci. 9: 2080-2102.

Kalaska, J.F., D.A.D. Cohen, M.J. Prud'homme, and M.L. Hyde. 1990. Parietal area 5 neuronal activity encodes movement kinematics, not movement dynamics. Exp. Brain Res. 80: 351-364.

Kano, M. 1996. A bridge between cerebellar long-term depression and discrete motor learning: Studies on gene knockout mice. Behav. Brain Sci. 19: 488-490.

Karst, G.M. and Z. Hasan. 1991. Initiation rules for planar, two-joint arm movements: Agonist selection for movements throughout the work space. J. Neurophysiol. 66: 1579-1593.

Kato, M. and M. Kimura. 1992. Effects of reversible blockade

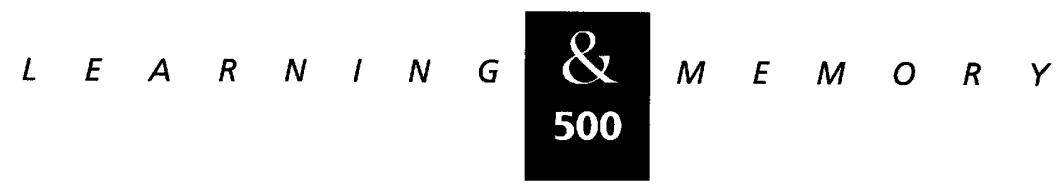


of basal ganglia on a voluntary arm movement. $J$. Neurophysiol. 65: 1516-1534.

Kawato, M. and H. Gomi. 1991. A computational model of four regions of the cerebellum based on feedback-error learning. Biol. Cybern. 68: 95-103.

Kettner, R.E., A.B. Schwartz, and A.P. Georgopoulos. 1988. Primate motor cortex and free arm movements to visual targets in three-dimensional space. III. Positional gradients and population coding of movement direction from various movement origins. J. Neurosci. 8: 2938-2947.

Kirsch, R.F. and W.Z. Rymer. 1987. Neural compensation for muscular fatigue: Evidence for significant force regulation in man. J. Neurophysiol. 57: 1893-1910.

Lacquaniti, F., E. Guigon, L. Bianchi, S. Ferraina, and R. Caminiti. 1995. Representing spatial information for limb movement: Role of area 5 in the monkey. Cereb. Cortex 5: 391-409.

Laporte, Y. and D.P.C. Lloyd. 1952. Nature and significance of the reflex connections established by large afferent fibers of muscular origin. Am. J. Physiol. 169: 609-621.

Lestienne, F. 1979. Effects of inertial load and velocity on the braking process of voluntary limb movements. Exp. Brain Res. 35: 407-418.

Llinás, R. 1981. Electrophysiology of the cerebellar networks. In Handbook of physiology. Section 1: The nervous system, Vol. II, part 2, Motor control (ed. F. Plum, V.B. Mountcastle, and S.R. Geiger), pp. 831-876. American Physiology Society, Bethesda, MD.

1989. Electrophysiological properties of the olivocerebellar system. In The olivocerebellar system in motor control (ed. P. Strata), pp. 201-208. Springer-Velag, New York, NY.

Llinás, R., R. Baker, and C. Sotelo. 1974. Electrotonic coupling between neurons in cat inferior olive. J. Neurophysiol. 37: 560-571.

Lloyd, D.P.C. 1943. Conduction and synaptic transmission of the reflex response to stretch in spinal cats. J. Neurophysiol. 6: $317-326$.

Mano, N., I. Kanazawa, and K. Yamamoto. 1989. Voluntary movements and complex-spike discharges of cerebellar purkinje cells. In The olivocerebellar system in motor control (ed. P. Strata), pp. 265-280. Springer-Verlag, New York, NY.

Marr, D. 1969. A theory of cerebellar cortex. J. Physiol. (Lond.) 202: 437-470.

Martin, J.H. and C. Ghez. 1991. Task-related coding of stimulus and response in cat red nucleus. Exp. Brain Res. 85: 373-388.

Matthews, P.B.C. 1981. Review lecture: Evolving views on the internal operation and functional role of the muscle spindle. J. Physiol. (Lond.) 320: 1-30.

Miall, R.C., M. Malkmus, and E.M. Robertson. 1996. Sensory prediction as a role for the cerebellum. Behav. Brain Sci. 19: $466-467$.

Millar, J. 1975. Flexion-extension sensitivity of elbow joint afferents in cat. Exp. Brain Res. 24: 209-214.

Nagasaki, H. 1989. Asymmetric velocity and acceleration profiles of human arm movements. Exp. Brain Research 74: 319-326.

Renshaw, B. 1941. Influence of discharge of motoneurones upon excitation of neighboring motoneurons. $J$. Neurophysiol. 4: 167-183.

1946. Central effects of centripetal impulses in axons of spinal ventral roots. J. Neurophysiol. 9: 191-204.

Robinson, C.J. and H. Burton. 1980. Organization of somatosensory receptive fields in cortical areas $7 \mathrm{~b}$, retroinsular postauditory and granular insula of $\mathrm{M}$. fascicularis. J. Comp. Neurol. 192: 69-92.

Robinson, F.R., J.C. Houk, and A.R. Gibson. 1987. Limb specific connections of the cat magnocellular red nucleus. J. Comp. Neurol. 257: 553-577.

Ryall, R.W. 1970. Renshaw cell mediated inhibition of Renshaw cells: Patterns of excitation and inhibition from impulses in motor axon collaterals. J. Neurophysiol. 33: $257-270$.

Ryall, R.W. and M. Piercey. 1971. Excitation and inhibition of Renshaw cells by impulses in peripheral afferent nerve fibers. J. Neurophysiol. 34: 242-251.

Sakurai, M. 1987. Synaptic modification of parallel fiber-Purkinje cell transmission in the in vitro guinea pig cerebellar slices. J. Physiol. (Lond.) 394: 463-480.

Shinoda, Y., Y. Sugiuchi, T. Futami, and R. Izawa. 1992. Axon collaterals of mossy fibers from the pontine nucleus in the cerebellar dentate nucleus. J. Neurophysiol. 67: 547-560.

Smith, A.M. 1981. The coactivation of antagonist muscles, Can. J. Physiol. Pharmacol. 59: 733-747.

1996. Does the cerebellum learn strategies for the optimal time-varying control of joint stiffness? Behav. Brain Sci. 19: 399-410.

Stein, J.F. and M. Glickstein. 1992. The role of the cerebellum in the visual guidance of movement. Physiol. Rev. 72: 967-1017.

Thach, W.T., H.P. Goodkin and J.G. Keating. 1992. The cerebellum and the adaptive coordination of movement. Ann. Rev. Neurosci. 15: 403-442.

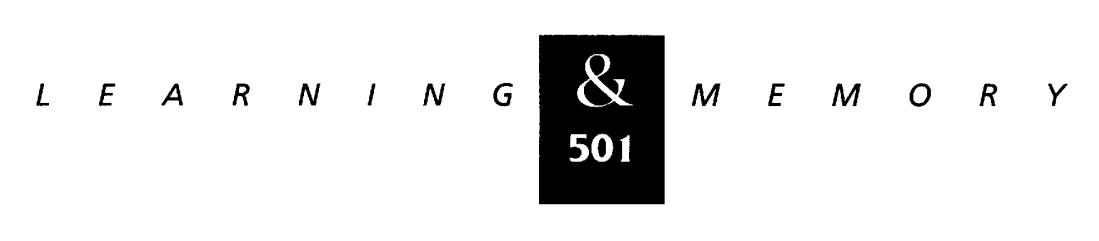


Tracey, D.J. 1979. Characteristics of wrist joint receptors in the cat. Exp. Brain Res. 34: 165-176.

Zatsirosky, V. and D. Seluyanov. 1983. The mass and inertia characteristics of the main segments of the human body. In Biomechanics VIIIB (ed. H. Matsui and K. Kobayashi), pp. 1152-1159. University Park Press, Champaign, IL.

Received February 14, 1997; accepted in revised form April 14, 1997.

\section{APPENDIX A: SIMULATION METHODS}

All simulations were performed using the classic Runge-Kutta fourth-order method or the Runge-Kutta-Nystrom method (Collatz 1966). Simultations were performed by integrating the dynamic system that defines the model described in Materials and Methods. The simulations were started with all of the system's variables initially set to 0.0, except for the PF-Purkinje cell connectivity matrix, which was set to the identity matrix.

For a typical single joint VITE-FLETE-CEREBELLUM simulation, the following equations were integrated: Equations 1-3 for VITE, equations 4-29 for FLETE, and equations 32-39 for the cerebellar model. The input variables were $G_{0}$, the Go signal multiplier, the target position vector $\left(T_{1}, T_{2}\right)$, and $P$, the coactivation signal; and the output variables were joint position $(\Theta)$ and velocities $(\dot{\Theta})$, muscle forces $\left(F_{1}, F_{2}\right), \alpha$-MN activities $\left(M_{1}, M_{2}\right)$, and spindle feedback $\left(E_{1}, E_{2}\right)$.

For the two-dimensional simulations, we used equations 1-3 for each of the two VITE systems (one for each limb segment), equations 4 and 5 , 9-31, and 40-51 for each FLETE system controlling the shoulder and the elbow joints, and equations $32-36,38$ and 39 for the cerebellar model extended to the two-dimensional case using the connectivity matrix depicted in Figure 10 . As in the single-joint simulation, the input variables were $G_{0}$, the GO signal multiplier; the target position vector $\left(T_{1}, T_{2}\right)$ for each VITE; GO signal multiplier common to both VITE systems; and the output variables were shoulder and elbow joint position $(\Theta$ and $\Phi$, respectively) and shoulder and elbow joint velocities $(\dot{\Theta}$ and $\dot{\Phi}$, respectively), muscle forces $\left(F_{1}, F_{2}\right)$, for the shoulder and elbow; $\alpha$-MN activities $\left(M_{1}, M_{2}\right)$ for the shoulder and elbow MN pools. Note that as the moment arm was set equal to 1.0, the shoulder and elbow torque variables in equations 40 and 41 corresponded actually to the difference between agonist and antagonist muscle forces fot the shoulder (equation 40) and elbow (equation 41) joints. Also, gravity was neglected as the simulations involved horizontal planar arm movements. 


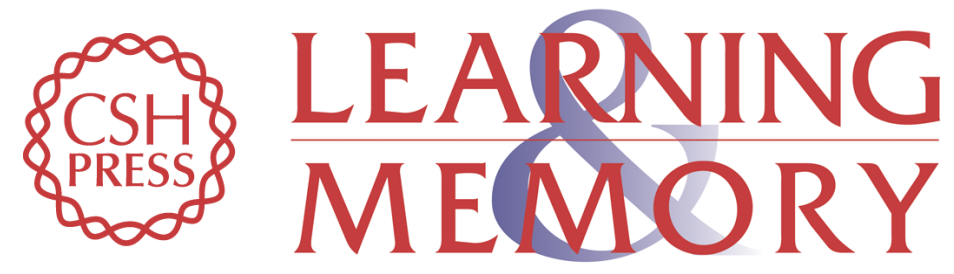

\section{A neural model of cerebellar learning for arm movement control: cortico-spino-cerebellar dynamics.}

J L Contreras-Vidal, S Grossberg and D Bullock

Learn. Mem. 1997, 3:

Access the most recent version at doi:10.1101//m.3.6.475

References This article cites 85 articles, 6 of which can be accessed free at: http://learnmem.cshlp.org/content/3/6/475.full.htmI\#ref-list-1

License

Email Alerting Receive free email alerts when new articles cite this article - sign up in the box at the Service top right corner of the article or click here. 